\title{
Pairs, sets and sequences in first-order theories
}

\author{
Albert Visser
}

Received: 30 May 2007 / Published online: 28 June 2008

(C) The Author(s) 2008

\begin{abstract}
In this paper we study the idea of theories with containers, like sets, pairs, sequences. We provide a modest framework to study such theories. We prove two concrete results. First, we show that first-order theories of finite signature that have functional non-surjective ordered pairing are definitionally equivalent to extensions in the same language of the basic theory of non-surjective ordered pairing. Second, we show that a first-order theory of finite signature is sequential (is a theory of sequences) iff it is definitionally equivalent to an extension in the same language of a system of weak set theory called WS.
\end{abstract}

Keywords Interpretations $\cdot$ Sequential theories $\cdot$ Weak set theory $\cdot$ Coding

Mathematics Subject Classification (2000) $\quad 03 \mathrm{~B} 30 \cdot 03 \mathrm{~F} 25 \cdot 03 \mathrm{~F} 40$

\section{Contents}

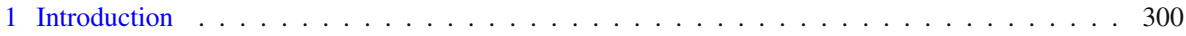

2 Definitions and basic facts . . . . . . . . . . . . . . . . . . . 301

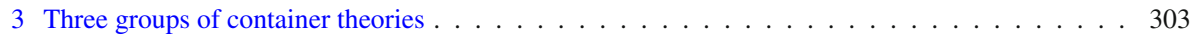

4 Adaptiveness . . . . . . . . . . . . . . . . . . . . . . . 310

5 Functional non-surjective ordered pairing . . . . . . . . . . . . . . . . . . . 313

6 The adaptiveness of weak set theory . . . . . . . . . . . . . . . . . . . . . 317

A. Visser $(\varangle)$

Department of Philosophy, Faculty of Humanities, Utrecht University,

Heidelberglaan 8, 3584 CS Utrecht, The Netherlands

e-mail: Albert.Visser@phil.uu.nl 


\section{Introduction}

This paper is a study of theories in predicate logic that possess "containers", like pairs, sets or sequences, for all objects of the theory. We explicate the possession of containers by a theory as direct interpretability of a suitable container theory. Here an interpretation is direct iff it is unrelativized and has absolute identity.

We prove, for two specific container theories that we have an alternative characterization of when a containee theory has the containers provided by the container theory. We show that, for these container theories, a containee theory has the corresponding containers iff it is definitionally equivalent to an extension in the same language of the container theory under consideration. Since definitional equivalence is the strictest notion of sameness of theories after extensional identity, we could almost say that in these cases the containee theories just are the extensions of the given container theory.

We will say that a theory $U$ is adaptive iff, whenever a theory $V$ directly interprets $U$, then $V$ is definitionally equivalent to an extension of $U$. With this notion in hand, we can rephrase our result: we will show of two specific container theories that they are adaptive. We will provide some further good properties of adaptive theories.

The two specific container theories that we will study are the theory of non-surjective, functional, ordered pairing and weak set theory (aka adjunctive set theory). The theories directly interpreting weak set theory are, modulo some minor details, the sequential theories. In a sequential theory $U$, we can reason about sequences of all objects in the domain of the theory. Using sequences, we can define partial truth predicates. Employing these truth predicates, we can prove restricted consistency statements for the theory $U$ relativized to appropriate definable cuts. As a consequence of these facts, one can prove many results concerning local and global interpretability for sequential theories.

The research reported in this paper has as "grand aim" finding coordinate free ways of thinking about such notions as sequentiality. We explicate "coordinate free" in terms of being defined in terms of an appropriate category of interpretations. Coordinate free properties will be preserved by isomorphisms in the chosen category. The coordinate free notion of adaptive theory studied in this paper is, admittedly, very far from fulfilling this grand aim. However, we submit, it constitutes a modest step in the right direction.

Thus, we have two motivations for studying adaptiveness. First, the fact that a theory $U$ is adaptive, provides us with a nice and perspicuous characterization of all containee theories possessing the containers provided by $U$. Second, the study of adaptiveness is interesting as the study of a coordinate free concept with good properties.

\section{Plan of the paper}

In Sect. 2, we introduce the basic concepts needed to understand the paper. Specifically, we give the basics of interpretations and interpretability. Section 3 is an introduction to container theories and their uses. In Sect. 4, we introduce the notion of adaptiveness and we prove some of its elementary properties. In Sect. 5, we show that the theory of 
functional, non-surjective, ordered pairing is adaptive. In Sect. 6, we show that a very weak set theory is adaptive.

\section{Definitions and basic facts}

We consider $\Delta_{1}^{\mathrm{b}}$-axiomatized theories in predicate logic of finite signature with identity. ${ }^{1}$ Officially, we demand that our theories have relational languages, i.e., that they lack function symbols and constants. However, we will be sloppy about this. Often we will speak about languages as if they have function symbols and constants. We assume implicitly that such function symbols and constants are eliminated using the well known translation algorithm of functional to relational languages.

\subsection{Translations and interpretations}

Let $\Sigma$ and $\Theta$ be finite signatures. A relative translation $\tau: \Sigma \rightarrow \Theta$ is given by a pair $\langle\delta, F\rangle$. Here $\delta$ is a $\Theta$-formula representing the domain of the translation. We demand that $\delta$ contains at most $v_{0}$ free. The mapping $F$ associates to each relation symbol $R$ of $\Sigma$ with arity $n$ an $\Theta$-formula $F(R)$ with variables among $v_{0}, \ldots, v_{n-1}$. Here identity is also included among the relation symbols of $\Sigma$. We translate $\Sigma$-formulas to $\Theta$-formulas as follows:

- $\left(R\left(y_{0}, \cdots, y_{n-1}\right)\right)^{\tau}:=F(R)\left(y_{0}, \cdots, y_{n-1}\right) ;^{2}$

- $(\cdot)^{\tau}$ commutes with the propositional connectives;

- $(\forall y A)^{\tau}:=\forall y\left(\delta(y) \rightarrow A^{\tau}\right)$;

- $(\exists y A)^{\tau}:=\exists y\left(\delta(y) \wedge A^{\tau}\right)$.

Suppose $\tau$ is $\langle\delta, F\rangle$. Here are some convenient conventions and notations.

- We write $\delta_{\tau}$ for $\delta$ and $F_{\tau}$ for $F$.

- We write $R_{\tau}$ for $F_{\tau}(R)$.

- We will always use "=" for the identity of a theory. In the context of translating, we will however switch to " $E$ ". So, $E_{\tau}$ is the translation of identity.

- We write $\mathbf{x}: \delta$ for: $\delta\left(x_{0}\right) \wedge \ldots \wedge \delta\left(x_{n-1}\right)$.

- We write $\forall \mathbf{x}: \delta A$ for: $\forall x_{0} \ldots \forall x_{n-1}(\mathbf{x}: \delta \rightarrow A)$.

- We write $\exists \mathbf{x}: \delta A$ for: $\exists x_{0} \ldots \exists x_{n-1}(\mathbf{x}: \delta \wedge A)$.

We can compose relative translations as follows:

$-\delta_{\tau \nu}:=\left(\delta_{\nu} \wedge\left(\delta_{\tau}\right)^{v}\right)$

$-R_{\tau v}=\mathbf{v}: \delta_{\tau v} \wedge\left(R_{\tau}\right)^{v}$.

We write $\nu \circ \tau:=\tau \nu$. Note that $\left(A^{\tau}\right)^{\nu}$ is provably equivalent in predicate logic to $A^{\tau \nu}$. The identity translation id $:=\operatorname{id}_{\Theta}$ is defined by:

\footnotetext{
1 The demand on the complexity of the axiom set is not as restrictive as it seems, since we often can diminish the complexity of the axiom set using versions of Craig's trick.

${ }^{2}$ Here $F(R)\left(y_{0}, \ldots, y_{n-1}\right)$ is our sloppy notation for: $F(R)\left[v_{0}:=y_{0}, \ldots, v_{n-1}:=y_{n-1}\right]$, the result of substituting the $y_{i}$ for the $v_{i}$. We assume that some mechanism for $\alpha$-conversion is built into our definition of substitution to avoid variable-clashes.
} 
$-\quad \delta_{\text {id }}:=\left(v_{0}=v_{0}\right)$,

$-\quad R_{\mathrm{id}}:=R\left(v_{0}, \ldots, v_{n-1}\right)$.

Note that translations as defined here only have good properties modulo provable equivalence. For e.g., $\delta_{\text {idoid }}=\left(v_{0}=v_{0} \wedge v_{0}=v_{0}\right)$, which is not strictly identical to $\delta_{\text {id. }}$

We say that a translation $\tau$ is unrelativized if the domain formula $\delta_{\tau}$ is $v_{0}=v_{0}$. A translation has absolute identity if it translates the identity relation to itself, i.o.w., $E_{\tau}=\left(v_{0}=v_{1}\right)$. A translation is direct if it is unrelativized and has absolute identity.

A translation $\tau$ supports a relative interpretation of a theory $U$ in a theory $V$, if, for all $U$-sentences $A, U \vdash A \Rightarrow V \vdash A^{\tau}$. (Note that this automatically takes care of the theory of identity. Moreover, it follows that $V \vdash \exists v_{0} \delta_{\tau}$.) We will write $K=\langle U, \tau, V\rangle$ for the interpretation supported by $\tau$. We write:

- $K: U \rightarrow V$ for: $K$ is an interpretation of the form $\langle U, \tau, V\rangle$,

- $K: V \triangleright U$ iff $K: U \triangleleft V$ iff $K: U \rightarrow V$. The $\triangleright$-notation is intended to be suggestive of interpretability as a generalization of derivability.

- $V \triangleright U$ iff $U \triangleleft V$ iff $\exists K K: V \triangleright U$.

If $M$ is an interpretation, $\tau_{M}$ will be its second component, so $M=\left\langle U, \tau_{M}, V\right\rangle$, for some $U$ and $V$.

Par abus de langage, we write " $\delta_{K}$ " for: $\delta_{\tau_{K}}$; " $R_{K}$ " for: $R_{\tau_{K}}$; " $A^{K}$ " for: $A^{\tau_{K}}$, etc.

Suppose $T$ has signature $\Sigma$ and $K: U \rightarrow V, M: V \rightarrow W$. We define:

$-\operatorname{id}_{T}: T \rightarrow T$ is $\left\langle T, \mathrm{id}_{\Sigma}, T\right\rangle$,

- $M \circ K: U \rightarrow W$ is $\left\langle U, \tau_{M} \circ \tau_{K}, W\right\rangle$.

We identify two interpretations $K, K^{\prime}: U \rightarrow V$ if:

$-\quad V \vdash \delta_{K} v_{0} \leftrightarrow \delta_{K^{\prime}} v_{0}$,

- $\quad V \vdash \mathbf{v}: \delta_{K} \rightarrow\left(R_{K} \mathbf{v} \leftrightarrow R_{K^{\prime}} \mathbf{v}\right)$.

One can show that, modulo this identification, the above operations give rise to a category INT of theories and interpretations. We mention two further salient interpretations. We say that a theory $V$ is an extension of a theory $U$, or $U \subseteq V$ if $U$ and $V$ have the same signature and the set of theorems of $U$ is a subset of the set of theorems of $V$. We can view extension as an interpretation $\mathcal{E}_{U V}$ with as underlying translation the identical translation. We say that a theory $V$ is an expansion of a theory $U$, if the signature of $V$ extends the signature of $U$ and the set of theorems of $U$ is a subset of the set of theorems of $V$. We can view extension as an interpretation $\varepsilon_{U V}$ with as underlying translation the identical translation.

If two theories are isomorphic in INT will be say that they are definitionally equivalent or synonymous. The monomorphisms of INT are precisely the faithful interpretations, i.e., the interpretations $\langle U, K, V\rangle$ such that, for all $U$-sentences $A$, we have $U \vdash A \Leftrightarrow V \vdash A^{K}$.

We say that an interpretation is unrelativized, has absolute identity, is direct, if its underlying translation is unrelativized, has absolute identity, is direct. The restriction of INT to direct interpretations is INT ${ }^{\text {dir }}$. It is easy to see that isomorphisms of INT are direct.

We will also meet interpretations with parameters. Since these only play a minor role in the paper, we treat them in Appendix A. 


\subsection{Multifunctions}

In this paper familiar notions like pairing will not generally be functional. This easily causes formulas to become unreadable, because one cannot use familiar notations. A lot of extra existential quantifiers are needed to compensate for the lack of the convenient notation for function composition. To diminish this awkwardness, we employ "multifunction notation". For some formulas $A \mathbf{x} y$, we introduce a notation of the form $F \mathbf{x} \cong y$. We use: $F t_{0} \ldots t_{n-1} \cong y$ for: $\exists y_{0}, \ldots, y_{n-1}\left(t_{0} \cong y_{0} \wedge \ldots \wedge t_{n-1} \cong\right.$ $y_{n-1} \wedge F y_{0} \ldots y_{n-1} \cong y$ ). We will only use this notation in formulas of the form $t \cong y$. We write $t \downarrow$ for: $\exists y t \cong y$.

If $\tau$ is a direct translation, we will write $F_{\tau} \mathbf{y} \cong x$ for: $(F \mathbf{y} \cong x)^{\tau}$.

\section{$2.3 U$-theories}

Consider any theory $U$. We say that a theory $V$ is a $U$-theory iff $U$ is directly interpretable in $V$. We say that $V$ is a parametric $U$-theory iff $U$ is directly parametrically interpretable in $V$. We have the following simple insight.

Theorem 1 The class of (parametric) $U$-theories is a subclass of the class of (parametric) $V$-theories iff $U$ directly (parametrically) interprets $V$. The classes of (parametric) $U$-theories and (parametric) $V$-theories coincide iff $U$ and $V$ are mutually directly (parametrically) interpretable.

Our intended application of the notion of $U$-theory is as an easy way to specify classes of theories having the containers provided by a given container theory. ${ }^{3}$ Suppose we want a theory $V$ to posses containers-like sets, pairs, sequences-for the elements of the domain of $V$. The presence of $U$ via direct interpretation provides these containers. We want to have containers for all elements of the domain of $V$, hence the container theory $U$ should be present via unrelativized interpretation. We want to have our containees unmodified, hence $U$ should be present via an interpretation with absolute identity.

\section{Three groups of container theories}

In this section, we introduce three salient groups of container theories. We present these groups in increasing order of strength.

\subsection{Theories of pairing}

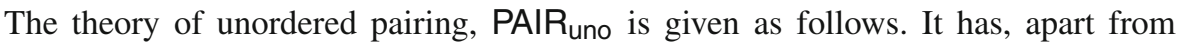
identity, one binary relation symbol $\in$. It has, apart from the axioms of identity, the following axiom.

$$
\text { pul } \vdash \forall u, v \exists x \forall y(y \in x \leftrightarrow(y=u \vee y=v)) .
$$

\footnotetext{
3 Of course, the notion of $U$-theory is far more general than this intended application.
} 
The theory non-surjective unordered pairing PAIR $\mathrm{Pno}_{\mathrm{ns}}$ is $\mathrm{PAIR}_{\mathrm{uno}}$ plus the axiom: pu2 $\vdash \exists x \forall y \quad y \notin x$.

The theory of ordered pairing PAIR $\mathrm{R}_{\mathrm{O}}$ is given as follows. It has, apart from identity, one ternary symbol pair. It has, apart from the axioms of identity, the following axioms.

po1 $\vdash \forall u, v \exists x$ pair $(u, v, x)$,

po2 $\vdash \forall u, v, u^{\prime}, v^{\prime}, x\left(\left(\operatorname{pair}(u, v, x) \wedge \operatorname{pair}\left(u^{\prime}, v^{\prime}, x\right)\right) \rightarrow\left(u=u^{\prime} \wedge v=v^{\prime}\right)\right)$.

The theory of non-surjective ordered pairing PAIR ${ }_{0, n s}$ is PAIR $_{\circ}$ plus the following axiom.

po3 $\vdash \exists x \forall u, v \neg \operatorname{pair}(u, v, x)$.

The theory of ordered pairing with at least two elements PAIR $_{0,2}$ is PAIR $_{\circ}$ plus the axiom $\vdash \exists x, y x \neq y$.

The theories PAIR $\mathrm{Pno}_{\text {and }}$ PAIR $\mathrm{P}_{\mathrm{O}}$ are mutually directly interpretable. We can interpret the unordered pairs in the ordered pairs by interpreting $x \in y$ as $\exists u$ (pair $(x, u, y) \vee$ pair $(u, x, y))$. We can interpret the ordered pairs in the unordered pairs using Kuratowski pairing. We interpret pair $(x, y, z)$ as:

$$
\begin{aligned}
\exists u, v \forall w( & (w \in z \leftrightarrow(w=u \wedge w=v)) \\
& \wedge(w \in u \leftrightarrow w=x) \\
& \wedge(w \in v \leftrightarrow(w=x \vee w=y))) .
\end{aligned}
$$

Similarly, for the theories PAIR $\mathrm{uno}_{\mathrm{n} \text {, ns }}$ and PAIR $\mathrm{P}_{0, \mathrm{~ns}}$. Thus, we can define the class

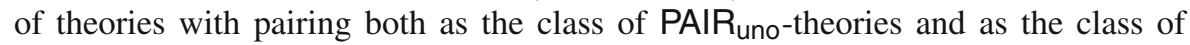
PAIR $_{0}$-theories. The non-surjective case of PAIR uno,ns $_{\text {and }} \mathrm{PAIR}_{\mathrm{o}, \mathrm{ns}}$ is similar.

The theory PAIR $_{0,2}$ is mutually directly parametrically interpretable with PAIR $_{\text {uno,ns }}$ and PAIR 0 ,ns. Thus, we can define the class of theories that parametrically have non-surjective pairing as the class of parametric PAIR $_{0,2}$-theories.

Each of our theories of pairing has a functional variant obtained by adding the functionality axiom for pairing. In the non-surjective case, we do not demand that there is a unique non-pair, but we add a constant 0 of which it is postulated that it is a non-pair. (Strictly speaking, we add a unary predicate, an axiom stating that it is inhabited by a unique object, and an axiom stating that every inhabitant is a non-pair.) We designate the functional variants using a superscript fun. For e.g., PAIR fun ${ }_{\mathrm{o} \text {,ns }}$ is the theory of functional non-surjective ordered pairing.

Example 1 We show that Robinson's Arithmetic $\mathrm{Q}$ is not a PAIR $\mathrm{O}_{0}$-theory, and, hence,

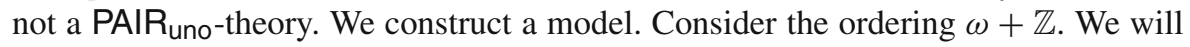
call the elements of the copy of $\omega$ : the finite cardinals. We will call the elements of the copy of $\mathbb{Z}$ : the infinite cardinals. We define:

- 0 is the least finite cardinal.

- Successor $S$ is the usual successor on the finite cardinals and is identity on the infinite cardinals.

- Addition + is the usual addition on the finite cardinals and is the maximum of its arguments if one of its arguments is infinite. Note that this gives us: $a \leq b \leftrightarrow$ $\exists c a+c=b$. 
- Multiplication $\times$ is defined as follows. We set $a \times b$ to be the usual product, if both $a$ and $b$ are finite. We take $a \times b:=0$, if one of $a, b$ is 0 . We take $a \times b=\max (a, b)$, if $0<\min (a, b)$ and one of $a, b$ is infinite.

Let $\sigma$ be the identity on the finite cardinals and the order successor on the infinite cardinals. It is easily seen that $\sigma$ is an automorphism of our model.

Suppose we could define in the arithmetical language a total multivalued pairing function $\langle\cdot, \cdot\rangle$. Suppose $a$ is an infinite cardinal and that $n$ is a finite cardinal. Suppose $\langle a, n\rangle \cong b$. If $b$ were finite we would have $\langle\sigma a, n\rangle \cong b$, which contradicts the assumption that $\langle\cdot, \cdot\rangle$ is a pairing function. So $b$ is infinite. Now note that $\left\langle\sigma^{z} a, n\right\rangle \cong \sigma^{z} b$, for all $z$ in $\mathbb{Z}$. It follows that every infinite cardinal instantiates a pair of the form $\left\langle\sigma^{z} a, 0\right\rangle$ and a pair of the form $\left\langle\sigma^{z^{\prime}} a, 1\right\rangle$. A contradiction.

Note that our model also satisfies associativity and commutativity of + and $\times$.

For some results concerning the complexity of theories of pairing, see [3], Chap. 8. W.V. Quine showed that, for a certain theory of pairing, say $U_{0}$, all $U_{0}$-theories are definitionally equivalent with theories with just one binary relation symbol. See Quine's paper [11]. We discuss Quine's result in somewhat more detail in Remark 3.

\subsection{Vaught theories of sets and sequences}

We first specify the theory VSEQ of Vaught sequences. To simplify the presentation of this theory and later ones, we introduce a fixed translation of theories of arithmetic. Let $U$ be any theory in the language of arithmetic. We assume that our language is the relational variant, with a unary predicate symbol $Z$ for zero, with a binary relation symbol $\mathrm{S}$ for successor, a binary symbol $<$ for the usual order, a ternary relation symbol $A$ for addition and a ternary relation symbol $M$ for multiplication. Let $\Theta$ be the signature of arithmetic, in its relational version, expanded with a unary relation symbol $\mathrm{N}$ and binary relation symbol $\mathrm{E}$. We translate the language of arithmetic to the new language of signature $\Theta$ by a translation \#, with $\delta_{\#}:=\mathrm{N}, E_{\#}:=\mathrm{E},{ }^{4} \mathrm{Z}_{\#}:=\mathrm{Z}$, $\mathrm{S}_{\#}:=\mathrm{S},<\#:=<, \mathrm{A}_{\#}:=\mathrm{A}, \mathrm{M}_{\#}:=\mathrm{M}$. We define $U^{\#}$ to be the theory axiomatized by the usual axioms for identity, by axioms $\vdash A^{\#}$, for every axiom $\vdash A$ of $U$ (including the axioms for identity), plus the axioms $\vdash P \mathbf{v} \rightarrow \mathbf{v}: \mathrm{N}$, where $P$ is any of the symbols $\mathrm{E}, \mathrm{Z}, \mathrm{S},<, \mathrm{A}, \mathrm{M}$.

We employ the arithmetical theory $R$ of [14], which is given by the following axioms. ${ }^{5}$

$$
\begin{aligned}
& \text { r1 } \vdash \underline{m}+\underline{n}=\underline{m+n}, \\
& \text { r2 } \vdash \underline{m} \times \underline{n}=\underline{m \times n}, \\
& \text { r3 } \vdash \underline{m} \neq \underline{n}, \text { if } m \neq n, \\
& \text { r4 } \vdash x<n \rightarrow \bigvee_{i<n} x=\underline{i}, \\
& \text { r5 } \vdash x<\underline{n} \vee x=\underline{n} \vee \underline{n} \leq x .
\end{aligned}
$$

\footnotetext{
4 Remember that we use " $E$ " as a generic name of identity. In contrast, "E" is a new constant distinct from "=" (aka " $E$ ") in $\Theta$. The symbol "E" will represent a congruence relation mimicking identity on the natural numbers.

5 Our version is in fact a variation that is definitionally equivalent to the formulation of [14].
} 
The theory VSEQ is obtained by expanding the signature $\Theta$ with a ternary symbol $\beta$. We will write $(x)_{y} \cong z$ for $\beta z y x$. The theory VSEQ is axiomatized by the following axioms.

vseq1 The axioms of $\mathrm{R}^{\#}$.

vseq2 $\vdash(x)_{y} \cong u \rightarrow y: \mathrm{N}$.

vseq3 $\vdash\left((x)_{y} \cong u \wedge y \mathrm{E} y^{\prime}\right) \rightarrow \forall v\left((x)_{y^{\prime}} \cong v \leftrightarrow u=v\right)$.

vseq4 For all $n$ and all sequences of variables $\mathbf{z}$ of length $n$, we have:

$\vdash \forall \mathbf{z} \exists x \bigwedge_{i<n}(x)_{i} \cong z_{i}$,

The class of VSEQ-theories was introduced by Robert Vaught in his [15]. We call these theories Vaught theories, following Pudlák in his [9]. Vaught's definition differs slightly from ours in that he works with a version of \# that translates identity to identity. Since Vaught's result also holds for our wider definition, we prefer the present definition.

Vaught proves the surprising theorem that every recursively enumerable Vaught theory of finite signature is axiomatizable by the embedded axioms of $\mathrm{R}$ plus one single axiom-scheme with one single binary schematic letter. As we will see, Vaught missed a simple argument that allows us to drop the embedded axioms of $\mathrm{R}$ from the theorem.

In his paper, Vaught introduces also another kind of theory: in our terms these are the VS-theories. The theory VS is a theory in the language with, apart from identity, one binary predicate $\in$. It is given by the axioms for identity plus the following axioms.

vs1 For each $n \in \omega$, we have:

$\vdash \forall x_{0}, \ldots, x_{n-1} \exists y \forall u\left(u \in y \leftrightarrow \bigvee_{i<n} u=x_{i}\right)$.

For VS-theories, Vaught's result is even better: every recursively enumerable VStheory of finite signature is axiomatizable by one single axiom-scheme with one single binary schematic letter. We show that the stronger result also holds for VSEQ-theories.

Clearly, we can define (non-extensional) pairing in VSEQ. Define:

$-x \in_{\tau} y: \leftrightarrow \exists u, v: \mathrm{N} \exists w\left(\langle u, w\rangle \cong y \wedge v<u \wedge(w)_{v} \cong x\right)$.

The numerical bound $u$ is added to keep undesired candidate elements out. It is easy to see that there is an interpretation based on $\tau$ witnessing the fact that VSEQ $\triangleright_{\text {dir }} V S$. So, Vaught's stronger result also holds for Vaught theories.

One can also show that VS $\triangleright_{\text {dir }}$ VSEQ. Regrettably I know of no place where the long verification is executed.

One can easily show that VS is locally directly interpretable in PAIR $\mathrm{uno}_{\text {,ns, }}$, i.o.w., PAIR $_{\text {uno,ns }}$ directly interprets every finite subtheory of VS. As the next theorem shows we cannot drop the locality.

Theorem 2 The theory VS is not interpretable in $\mathrm{PAIR}_{\mathrm{uno}, \mathrm{ns}}$

Proof Since VS interprets the theory R of [14], we find that VS is essentially undecidable. On the other hand it is well known that there are decidable extensions of PAIR uno,ns. (see e.g., [3] and [2]) 


\subsection{Sequentiality}

In this subsection, we introduce the notion of sequential theory.

\subsubsection{Weak set theories and theories of sequences}

Let $U$ be any arithmetical theory, i.e., a theory in the language of arithmetic that extends $Q$. We define a theory $\operatorname{PSEQ}(U)$ in the signature $\Theta^{+}$, which is the signature $\Theta$ of VSEQ extended with a binary predicate seq. We will write $0 \cong x$, for $Z x$, $\mathrm{S} x \cong y$ for $\mathrm{S} x y,(x)_{y} \cong z$ for $\beta z y x$.

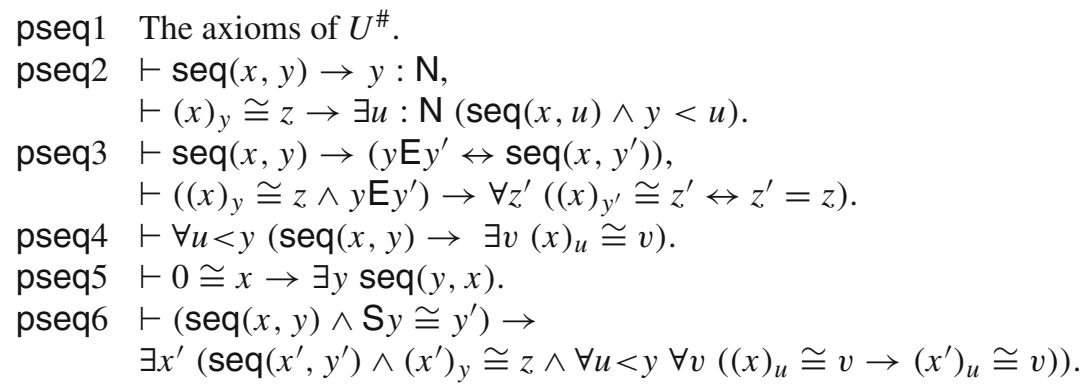

Let us say that an arithmetical theory is absolutely weak if it interpretable in Robinson's Arithmetic Q just by relativization to a definable class that is closed under the arithmetical operations and downwards closed w.r.t. <. Such theories as $Q, \mathrm{~S}_{2}^{1}$, $I \Delta_{0}+\Omega_{1}$ and $I \Delta_{0}+\Omega_{17}+\Sigma_{1}$-collection are absolutely weak. Any $\Pi_{1}^{0}$-axiomatized theory interpretable in $\mathrm{Q}$ is absolutely weak, and so is any $\Pi_{1}^{0}$-axiomatized theory interpretable in $\mathrm{Q}$ plus $\Sigma_{1}$-collection. For e.g., $\mathrm{Q}+$ incon $(\mathrm{Q})$ is interpretable in $\mathrm{Q}$, but not absolutely weak. We have:

Theorem 3 If $U$ and $V$ are absolutely weak then PSEQ $(U)$ and PSEQ $(V)$ are mutually directly interpretable.

The main point here is of course that we may restrict the interpretation of $\mathrm{N}$, while keeping the general object domain unrelativised. We can improve the theorem further by adding operations like concatenation of sequences, restriction of sequences, insertion of elements in sequences and the like. For the main ingredients of the proof see [4] or [9].

Just as in the case of Vaught theories, there is a closely related set theory. We define the container theory WS, weak set theory or adjunctive set theory, as follows. This theory is a theory in the language with just identity and one binary predicate symbol $\in$. It is given by the following axioms.

ws1 $\vdash \exists x \forall y \quad y \notin x$,

ws2 $\vdash \forall u, v \exists x \forall y(y \in x \leftrightarrow(y \in u \vee y=v))$.

We define the following multifunctions:

$-\emptyset \cong x: \leftrightarrow \forall y \quad y \notin x$,

$-\mathrm{S}_{v} u \cong x: \leftrightarrow \forall y(y \in x \leftrightarrow(y \in u \vee y=v))$. 
We can now rephrase our axioms simply as: $\vdash \varnothing \downarrow$ and $\vdash S_{v} u \downarrow$. We have the following remarkable theorem.

Theorem 4 Suppose $T$ is absolutely weak. Then, PSEQ $(T)$ is mutually directly interpretable with WS.

We provide some pointers for the proof of Theorem 4 in Appendix B.

A theory is sequential iff it is a WS-theory. A theory is parametrically sequential iff it is a parametric WS-theory. Alternatively, sequential theories are precisely the $\operatorname{PSEQ}(T)$-theories for any absolutely weak $T$.

Clearly, the theory WS extends VS. The next theorem shows that WS is essentially stronger than VS.

\section{Theorem 5 VS does not interpret WS.}

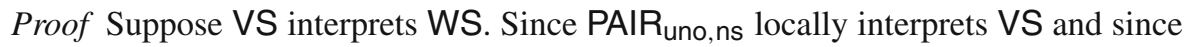
WS is finitely axiomatized, it follows that PAIR $\mathrm{uno}_{\mathrm{u}, \mathrm{ns}}$ interprets WS. But this is impossible, since WS is essentially undecidable (e.g., since it interprets $Q$ ) and PAIR $R_{u n o, n s}$ has a decidable extension.

So we have: PAIR uno,ns $\triangleleft_{\text {dir }}$ VS $\triangleleft_{\text {dir }}$ WS, and this sequence is strictly ascending.

In Appendix B, we provide some historical notes on the development of the notion of sequentiality.

\subsubsection{Examples, small facts and questions}

We first provide some examples to illustrate that sequentiality is not preserved by mutual interpretability.

Example 2 One can show that no consistent theory of the form $U \oplus U$ is sequential. ${ }^{6}$ Suppose $U$ is consistent and sequential. Then $U$ is mutually interpretable with $U \oplus U$, but $U \oplus U$ is not sequential.

Example 3 The theory $\mathrm{Q}$ interprets $\mathrm{WS}$, but not directly, since $\mathrm{Q}$ is not even a PAIR $_{\text {uno-theory. See Example } 1 .}$

Question 1 Is there a sequential arithmetical theory that is minimal w.r.t. direct interpretability?

The following fact implies that WS does not interpret $Q$ via an interpretation that preserves identity.

Fact 1 No constants are definable in WS, in other words, there is no formula Ax with at most $x$ free, such that WS $\vdash \exists ! x$ Ax.

\footnotetext{
6 The operation $\oplus$ is the sum in INT. For a definition of a specific choice of this operation on theories, see [21].
} 
Proof It is clearly sufficient to produce a model of WS with an automorphism without fixed points. Let $\mathcal{N}$ be any countable model of WS. We may assume that the domain of $\mathcal{N}$ is the set of natural numbers. Let $\tau$ be the mapping that interchanges $2 n$ and $2 n+1$, for every $n$. We define a new model, $\mathcal{N}^{\star}$, as follows:

$-\quad x \in^{\star} y: \leftrightarrow \exists z((y=2 z \wedge x \in z) \vee(y=2 z+1 \wedge \tau x \in z))$.

If we use [·] for the entire function, we can rewrite this as: $x \in^{\star} y: \leftrightarrow \tau^{y} x \in\left[\frac{y}{2}\right]$. It is easily seen that $\mathcal{N}^{\star}$ is again a model of WS. Note that $\tau x \in^{\star} \tau y$ iff $\tau^{y} x=\tau^{\tau y} \tau x \in$ $\left[\frac{\tau y}{2}\right]=\left[\frac{y}{2}\right]$. Thus, $\tau$ is an automorphism of $\mathcal{N}^{\star}$ with no fixed points.

Question 2 It is well known that every sequential theory is mutually locally interpretable with an arithmetical theory. Is it also true that every sequential theory is mutually interpretable with an arithmetical theory?

We provide an example of a parametrically sequential theory that is not sequential.

Example 4 We consider the theory $U$ with a ternary predicate $x \in_{z} y$. The axioms of $U$ are obtained from those of WS by replacing subformulas of the form $x \in y$ by $x \in_{z} y$. (Here $z$ does not occur in the original axioms and obtains a universal reading in the axioms as a whole). Trivially $U$ is parametrically sequential.

To see that $U$ is not sequential, consider any countable model $\mathcal{M}$ of WS. We may assume that the domain of $\mathcal{M}$ consists of the integers. We define $a \in_{c} b: \leftrightarrow(a+c) \in$ $(b+c)$, thus obtaining a model $\mathcal{N}$ of $U$. Since for every $a$ and $b$ in the domain of $\mathcal{N}$ there is an automorphism that sends $a$ to $b$, to wit $(\cdot)+(b-a)$, every $\mathcal{N}$-definable class is either empty or the universe. Thus, there can be no interpretation of WS in $U$, since e.g., the class of empty sets cannot be defined.

Remark 1 In this paper, we do not consider multidimensional interpretations. We point out here that under the most obvious reading of the notion of direct multidimensional interpretation, we can replace a parametric interpretation of WS by a non-parametric multidimensional one. For e.g., suppose that $\langle K, A\rangle: V \triangleright \mathrm{WS}$ is a one-dimensional interpretation with one parameter. We can replace $\langle K, A\rangle$ by a two-dimensional one $M$ by setting:

- $(x, y) \in_{M}(u, v): \leftrightarrow A(u) \wedge \exists w\left(\langle x, y\rangle_{K(u)} \cong w \wedge w \in_{K(u)} v\right)$.

As usual, we employ Kuratowski pairing: $\langle x, y\rangle_{K(u)}:=\{\{x\},\{x, y\}\}_{K(u)}$.

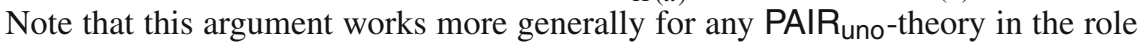
of WS.

Remark 2 In our framework, all theories were supposed to have identity. However, it is nice to reduce the signature to just one binary relation without identity. For example, we can easily show that modulo definitional equivalence identity can be eliminated from WS. Define:

- $x E^{*} y: \leftrightarrow \forall u(u \in x \leftrightarrow u \in y) \wedge \forall v(x \in v \leftrightarrow y \in v)$

Let WS* be the theory WS, in the language without identity, where we replace $=$ in the non-logical axioms by $E^{*}$. We claim that WS* and WS are definitionally equivalent. 
The interpretation $K$ of WS* in WS is the identical one. The interpretation $M$ of WS in WS* is by interpreting $=$ by $E^{*}$. The correctness of $K$ follows from the fact that WS $\vdash \forall v(x \in v \leftrightarrow y \in v) \rightarrow x=y$ and hence WS $\vdash \forall x, y\left(x E^{*} y \leftrightarrow x=y\right)$. The correctness of $M$ is easy, the main part being the verification of the identity axioms for $E^{*}$. We have that $E_{M}^{K}=E^{*}$ and WS $\vdash x E^{*} y \leftrightarrow x=y$. Moreover, $M \circ K$ is the identity interpretation on WS* Thus, WS and WS* are definitionally equivalent.

\section{Adaptiveness}

We say that a theory $U$ is adaptive iff every $U$-theory is definitionally equivalent with an extension of $U$. (The theory $U$ is such that every $V$ that directly interprets $U$ can be "adapted" to a theory that extends $U$ ).

In [22], it is shown that a morphism in INT is an epimorphism iff it can be split into an extension followed by an isomorphism. Thus, a theory $U$ is adaptive if and only iff, for all $V$, if $U \triangleleft_{\text {dir }} V$, then $U \triangleleft_{\mathrm{epi}} V$. Epimorphisms have the same characterization in INT ${ }^{\text {dir }}$ as in INT. Hence, adaptiveness is definable in terms of INT ${ }^{\text {dir }}$. This shows that adaptiveness is coordinate free when we have INT ${ }^{\text {dir }}$ as our background category.

Question 3 Is adaptiveness definable in terms of INT?

The next theorem shows that the property of adaptiveness is a "good property" w.r.t. the category INT.

Theorem 6 Suppose $U$ is adaptive and $V$ is definitionally equivalent to $U$. Then $V$ is adaptive.

Proof Suppose $U$ is adaptive and $U$ is definitionally equivalent with $V$. Let $K$ and $K^{-1}$ witness the isomorphism of $U$ and $V$. Let $M: V \rightarrow V^{\prime}$ be direct. Since isomorphisms are direct, it follows that $M \circ K: U \rightarrow V^{\prime}$ is direct. $^{7}$

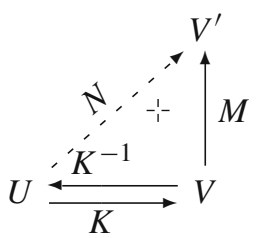

Hence, there is an epimorphism $N: U \rightarrow V^{\prime}$. We may conclude that $N \circ K^{-1}: V \rightarrow$ $V^{\prime}$ is also an epimorphism.

Question 4 Consider the degree structures DEG ${ }^{\text {dir }}$ of direct interpretability and $D E G^{e p i}$ of epimorphic interpretability. Does the embedding functor from DEG ${ }^{\text {epi }}$ into DEG ${ }^{\text {dir }}$ have a left adjoint? (The elements in the image of such a functor would be precisely the adaptive theories.) If the answer is no, can we find suitable restrictions of the degree structures for which the answer is yes?

\footnotetext{
7 The small cross in the middle of the diagram tells us that the diagram does not necessarily commute.
} 
The next theorem gives a very modest "lower bound" result for adaptiveness. Let INF be the theory, in the language of identity, stating, for every $n$, that there are at least $n$ elements.

\section{Theorem 7 Every adaptive theory contains INF.}

Proof Let $U$ be an adaptive theory and suppose that $U$ has a model of $n$ elements. Let $P$ be a 0 -ary predicate not in the signature of $U$. Let $U^{P}$ be the theory we obtain from $U$ by expanding the signature of $U$ with $P$, adding no extra axioms. Clearly, $U^{P} \triangleright_{\text {dir }} U$. Let $W$ be an extension of $U$ that is definitionally equivalent to $U^{P}$. Suppose $U$ has $N$ models (modulo isomorphism) on $n$ elements. By assumption $N>0$. Then, $U^{P}$ has $2 N$ models on $n$ elements. Hence, $W$ has $2 N$ models on $n$ elements. But every model of $W$ is ipso facto a model of $U$. A contradiction.

It follows, e.g., that predicate logic is not adaptive. The next question concerns the possibility of a better lower bound.

Question 5 Does every adaptive theory directly interpret PAIR $\mathrm{uno}_{\text {? }}$

The next example illustrate that rather strong theories need not be adaptive.

Example 5 In this example we show that PA is not adaptive. ${ }^{8}$ Let $\mathrm{PA}^{P}$ be Peano Arithmetic with an additional propositional variable $P$. We add no further axioms regarding this variable. Clearly, $\mathrm{PA}$ is directly interpretable in $\mathrm{PA}^{P}$. Suppose PA is adaptive. Then, there is an extension $T$ of PA, such that $T$ is definitionally equivalent to $\mathrm{PA}^{P}$. We work in $\mathrm{PA}^{P}$.

Let $K: T \rightarrow \mathrm{PA}^{P}$ be the isomorphism from $T$ to $\mathrm{PA}^{P}$. Let $M$ be its inverse. Using Dedekind's construction of an isomorphism of two models of second order arithmetic, we can now build, $\mathrm{PA}^{P}$-verifiably, a definable isomorphism $F$ between the $\mathrm{PA}^{P}$-numbers (the domain of the theory) and $K$-numbers (the domain of the interpretation $K$ ). ${ }^{9}$ To verify that we did define an isomorphism, we need the fact that we have full induction both for the $\mathrm{PA}^{P}$-numbers and the $K$-numbers. It follows from the existence of $F$, that, for arithmetical sentences $C:(\dagger) \mathrm{PA}^{P} \vdash C \leftrightarrow C^{K}$.

But now we have $\mathrm{PA}^{P} \vdash P \leftrightarrow P^{M K}$, since $M$ and $K$ are each others inverses. From the fact that $P^{M}$ is arithmetical, it follows, by $(\dagger)$, that $\mathrm{PA}^{P} \vdash P \leftrightarrow P^{M}$. Quod impossible, because $P^{M}$ is arithmetical.

We provide a necessary condition for adaptiveness.

Theorem 8 Suppose $U$ is consistent and adaptive. Then, $U$ has a finitely axiomatized, essentially undecidable extension, which is complete $\Sigma_{1}^{0}$. Moreover $U$ is itself complete $\Sigma_{1}^{0}$.

Proof Suppose $U$ is consistent and adaptive. We show that:

$$
V:=\left(I \Sigma_{2}+\operatorname{con}(U+\mathrm{INF})\right) \nabla_{\operatorname{dir}} U \text {. }
$$

\footnotetext{
8 The fact that PA is not adaptive also follows from Theorem 8 .

9 Note that $F$ is an isomorphism between interpretations, and that $K$ is an isomorphism between theories. For elaboration of such ideas, see [21].
} 
(We have shown that $U$ implies INF. We need, however, INF to be verifiably present.) We first construct an interpretation using the Henkin-Feferman construction. Note that the proof that the Henkin-Feferman construction yields an infinite path is precisely a $\Sigma_{2}$-induction. Moreover, both the "truth predicate" and the domain $\delta$ provided by the Henkin-Feferman construction are $\Delta_{2}^{0}$. So the equivalence relation $E$ between the elements $\delta$ is also $\Delta_{2}^{0}$. Since $U$ extends INF, we can "collapse" the domain to the natural numbers assigning to each equivalence class precisely one number. The collapsing function on $\delta$ can be defined as follows. We say that a sequence $\sigma$ is accepting iff (a) $\sigma_{j}$ is in $\delta$ for each $j<\operatorname{length}(\sigma)$, and (b) $\sigma_{j}<\sigma_{k}$ and $\neg\left(\sigma_{j} E \sigma_{k}\right)$, for $j<k<$ length $(\sigma)$, and (c) for all $y \leq \sigma_{\text {length }(\sigma)-1}$ there is a $j<\operatorname{length}(\sigma)$ such that $\sigma_{j} E y$. We prove, by $\Sigma_{2}$-induction that for every $i$ there is an accepting sequence of length $i$. Note that this uses the fact that INF is verifiably present. We set $F x=i$ iff there is an accepting sequence $\sigma$ with $\sigma_{i} E x$. It is easy to verify that $F$ has the desired properties. Finally, we use $F$ to transform our interpretation to a direct one.

Let $U^{\prime}$ be an extension of $U$ that is definitionally equivalent with $V$. Since finite axiomatizability is preserved by definitional equivalence, we may take $U^{\prime}$ to be finitely axiomatized. Moreover, essential undecidability is also preserved under definitional equivalence. Finally, $V$ is complete $\Sigma_{1}^{0}$, hence, so is $U^{\prime}$. Since $U^{\prime}$ is finitely axiomatized, it follows that $U$ too is complete $\Sigma_{1}^{0}$.

Note that it does not follow that adaptive theories are themselves essentially undecidable. Theorem 8 implies that PA and ZF (and, more generally, all essentially reflexive theories) are not adaptive, since these theories have no finitely axiomatized extensions.

We provide a simple example of a group of adaptive theories.

Example 6 Let $V$ be any PAIR uno,ns-theory. Let $U:=V^{P}$ be the theory $V$, expanded with a unary predicate $P$, without any further axioms concerning $P$. We show that $U$ is adaptive.

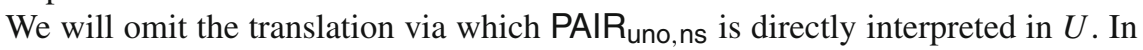
$U$ we define:

$-\langle x, y\rangle:=\{\{x\},\{x, y\}\}$,

$-0 \cong y: \leftrightarrow \forall z z \notin y$,

- $\mathrm{S} x:=\langle x, 0\rangle$,

$-\quad \underline{0}:=0, \underline{n+1}:=\mathrm{S} \underline{\underline{n}}$,

-[]$_{0}:=0,[\mathbf{x}, y]_{n+1}:=\left\langle[\mathbf{x}]_{n}, y\right\rangle$,

As usual we can verify that $\langle\cdot, \cdot\rangle$ is a pairing, that 0 is no successor, that $S$ is total and injective, that the $\underline{n}$ are pairwise disjoint, and that the $[\mathbf{x}]_{n}$ have the properties of sequences of length $n$.

Suppose $K: W \triangleright_{\text {dir }} U$. We order the predicates of $W$ as $Q_{i}$ with arity $a_{i}$. Let $\alpha$ be the translation on which $K$ is based. We define a new direct translation $\beta$ as follows:

- $\quad R_{\beta} \mathbf{x}: \leftrightarrow R_{\alpha} \mathbf{x}$, if $R$ is a $U$-predicate unequal to $P$.

$-P_{\beta} x: \leftrightarrow \bigvee_{i} \exists \mathbf{y}\left(\left\langle\underline{i},[\mathbf{y}]_{a_{i}}\right\rangle_{\alpha} \cong x \wedge Q_{i} \mathbf{y}\right)$.

We translate the language of $W$ in the language of $U$ via the direct translation $\tau$ given by:

$-\quad Q_{i, \tau} \mathbf{y}: \leftrightarrow \exists x\left(\left\langle\underline{i},[\mathbf{y}]_{a_{i}}\right\rangle \cong x \wedge P x\right)$. 
It is easy to see that $W \vdash\left(Q_{i} \mathbf{y}\right)^{\tau \beta} \leftrightarrow Q_{i} \mathbf{y}$. Define:

$-\quad T:=U+\left\{A^{\tau} \mid A\right.$ is an axiom of $\left.W\right\}$ $+\left\{\forall \mathbf{x}\left(R x \leftrightarrow(R \mathbf{x})^{\beta \tau}\right) \mid R\right.$ is a predicate of $\left.U\right\}$.

It is now easy to see that $\langle W, \tau, T\rangle$ and $\langle T, \beta, W\rangle$ form an isomorphism.

The above example gives us immediately the following theorem, which tells us that (consistent) adaptive theories can be arbitrarily strong.

Theorem 9 Consider any consistent theory $U$, which contains INF. Then, there is an adaptive consistent $V$ such that $V \triangleright_{\operatorname{dir}} U$.

In Sect. 5, we show that PAIR fun is adaptive. This leaves many loose ends.

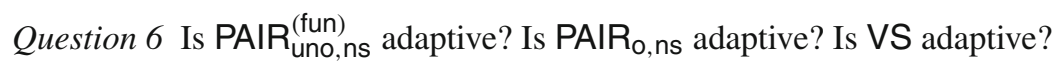

Remark 3 We briefly comment on the relation between Question 6 and the result by Quine in his paper [11]. It is not very clear what precisely Quine proves. His own statement is just that every first-order model is an internal model (with absolute identity) of another first-order model for the signature of just one binary predicate symbol. His construction is not the construction of an INT-isomorphism, because it allows extension of the domain and because it involves the use of standard numbers. Inspecting the argument, one sees that the domain extension can be avoided. It is needed to add pairs to the original model. We do not need it if we stipulate the pairs to be already present. Second, the standard numbers only appear because Quine allows infinite signatures. If we only consider finite signatures this feature disappears too.

Thus, upon inspection, we can extract the following result. Let PAIR $\mathrm{unn}_{\mathrm{uno}, \mathrm{q}}$ be the theory given by PAIR fun plus the following axioms: $\vdash x \neq\{x, y\}$ and $\vdash x \neq\{\{x\}, y\} .{ }^{10}$ Then, Quine's proof gives (for finite signatures!): every PAIR $\mathrm{fun}_{\mathrm{uno}} \mathrm{q}^{\text {-theory is definition- }}$ ally equivalent with a theory in the language with just one binary relation symbol.

I conjecture that it should be possible to replace the use of PAIR $R_{u n o, q}^{\text {fun }}$, by PAIR uno,ns $_{\text {, }}$, using methods analogous to those in the proof of Lemma 1.

\section{Functional non-surjective ordered pairing}

We prove that PAIR fun is adaptive. Some of the ideas of our argument are taken from a proof by Ferrante, Rackoff and Hossley, that PAIR ${ }_{0,2}^{\text {fun }}$ is undecidable. ${ }^{11}$ (see [3], pp 165-169)

We work, for the moment, in PAIR ${ }_{\mathrm{o}, \mathrm{ns}} .{ }^{12}$ We define:

- $\operatorname{Pair}(u): \leftrightarrow \exists v, w \operatorname{pair}(v, w, u)$,

10 This last axiom is slightly stronger than Quine's version, which is $\vdash x \neq\{\{x\}\}$. However, I think that Quine's proof needs the stronger variant.

11 This result was first proved by Hanf and Morley.

12 We think that it is of some independent interest that Lemma 1 also works in the absence of functionality. 
$-\quad 0 \cong u: \leftrightarrow \neg$ Pair $(u) .^{13}$

$-\langle u, v\rangle \cong w: \leftrightarrow \operatorname{pair}(u, v, w)$,

$-\pi_{i} w \cong u_{i}: \leftrightarrow \exists u_{1-i}\left\langle u_{0}, u_{1}\right\rangle \cong w$,

- $\mathrm{s} u:=\langle 0, u\rangle$.

- $\widetilde{0}:=0, \widetilde{n+1}:=\mathrm{s} \tilde{n}$,

- $u: D_{0}: \leftrightarrow \exists v\langle 0, v\rangle \cong u, u: D_{0}^{+}: \leftrightarrow 0 \cong u \vee u: D_{0}$,

$-\langle v\rangle_{1}:=v,\langle v, \mathbf{w}\rangle_{n+2}:=\left\langle v,\langle\mathbf{w}\rangle_{n+1}\right\rangle$.

We will often suppress the numerical indices of our sequence coding. Note that the same object may code sequences of different lengths. An important insight is that $\mathrm{PAIR}_{\mathrm{o}, \mathrm{ns}} \vdash x \neq y \rightarrow\langle x, \mathbf{u}\rangle \neq\langle y, \mathbf{v}\rangle$. We will also suppress the tildes above our numerals. Note that, for numbers $i \neq j$, we have PAIR $\mathrm{P}_{\mathrm{o}, \mathrm{ns}} \vdash \neg(i \cong u \wedge j \cong u)$. Moreover, we have: PAIR $\mathrm{R}_{\mathrm{O}, \mathrm{ns}} \vdash i+1 \cong u \rightarrow u: D_{0}$.

Here is the heuristics of our argument. Consider a theory $U$ that directly interprets PAIR $R_{\mathrm{o}, \mathrm{ns}}^{\text {fun }}$. Using the given pairing we want to redefine it in such a way that the new pairing contains all information about the predicates of $U$. Our strategy is to regain the predicate $P_{j}$ via: $P_{j} \mathbf{z} \leftrightarrow \exists v\langle\langle j+1, \mathbf{z}\rangle, v\rangle=v$. In fact every step in the proof follows from this basic design plan. To avoid unwanted cases of $\langle\langle j+1, \mathbf{z}\rangle, v\rangle=v$ we need the following property for our original pairing: $(\dagger) \vdash \neg\langle u, v, w\rangle \cong w$. However, PAIR fun does not provide $(\dagger)$. So, first we directly interpret PAIR $_{\mathrm{o}, \mathrm{ns}}^{\text {fun }}$ plus $(\dagger)$

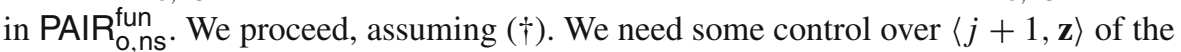
new pairing. To achieve this, we use $D_{0}$ as a "safe domain" on which the new pairing we are constructing behaves in well understood ways. We employ isomorphic copies of the predicates of $U$ on $D_{0}$. We then construct the desired pairing for the isomorphic copies on $\mathrm{t} D_{0}$. To regain the original properties on the full domain from their copies, we need to code the isomorphism between the full domain and $D_{0}$ in the new pairing. This isomorphism is the successor function defined using the old pairing.

We first interpret $(\dagger)$. It turns out that we can do this without the extra assumption of functionality.

Lemma 1 We have: $\operatorname{PAIR}_{\mathrm{o}, \mathrm{ns}}^{\text {(fun) }} \triangleright_{\mathrm{dir}}\left(\operatorname{PAIR}_{\mathrm{o}, \mathrm{ns}}^{\text {(fun) }}+\forall u, v, w \neg\langle u, v, w\rangle \cong w\right)$.

Proof The proof is a diagonal argument. We first give our proof for the non-functional case. We provide a direct interpretation $P$ with underlying translation $\varpi$ in $\mathrm{PAIR}_{\mathrm{O}, \mathrm{ns}}$ of the theory $\operatorname{PAIR}_{\mathrm{o}, \mathrm{ns}}+\forall u, v, w \neg\langle u, v, w\rangle \cong w$. We define:

$-\langle u, v\rangle_{\varpi}:=\left\{\begin{array}{l}\langle 1, u, v\rangle \text { if } \exists w, y, z(\langle w, y, z\rangle \cong v \wedge\langle 0, u, v\rangle \cong z) . \\ \langle 0, u, v\rangle \text { otherwise }\end{array}\right.$

It is easy to see we did define a non-surjective pairing. Suppose $\langle a, b, c\rangle_{\varpi} \cong c$, say $\langle b, c\rangle_{\varpi} \cong d$ and $\langle a, d\rangle_{\varpi} \cong c$. By the definition, for some $i, j$ in $\{0,1\}$, we have $\langle j, b, c\rangle \cong d$ and $\langle i, a, d\rangle \cong c$. Note that $i$ and $j$ are uniquely determined. Suppose $i=0$. Then, by the first clause of the definition of $\varpi$, we find $i=1$. A contradiction. So, we have $i=1$. Thus, the first clause must be active and we have $\langle 0, a, d\rangle \cong c$.

\footnotetext{
13 Remember that, by our convention for $\cong$, this notation does not presuppose that there is a unique non-pair. In case we are working in PAIR fun $\mathrm{O}_{\mathrm{ns}}$, the definiens may be replaced by $0=u$.
} 
(Note that $z$ in the first clause is uniquely determined by $v$.) But then we do not have $\langle 1, a, d\rangle \cong c$. A contradiction. So, we cannot have $\langle a, b, c\rangle_{\varpi} \cong c$.

Note that, if our original pairing is functional, then the new pairing constructed is also functional. Here, of course, we use the constant 0 in stead of the defined 0 of the non-functional case. So, we also have our result for functional pairing.

We turn to our main construction. We work with functional pairing. We use the explicitly added constant 0 in stead of the defined 0 , also in the previous definitions. Suppose $U \triangleright_{\text {dir }}$ PAIR o,ns $_{\text {fun }}$ By Lemma 1 , we can find a $K$ such that

$$
K: U \triangleright_{\text {dir }}\left(\mathrm{PAIR}_{\mathrm{O}, \mathrm{ns}}^{\mathrm{fun}}+(\dagger)\right)
$$

Let the underlying translation of $K$ be $\tau$. In the context of $U$ we will suppress the subscripts and superscripts $\tau$. We define a "safe" isomorphic copy of $U$ inside $U$, via the translation $\mu$, as follows.

- $\delta_{\mu}:=D_{0}$ (remember that $D_{0}$ is the class of successors),

- $P_{\mu} \mathbf{v}: \leftrightarrow \exists \mathbf{w}\left(\bigwedge_{i} \mathrm{~s} w_{i}=v_{i} \wedge P \mathbf{w}\right)$.

Let $M: U \triangleright U$ be the interpretation based on $\mu$. Clearly, $\mathrm{s}$ is an isomorphism between $\mathrm{id}_{U}$ and $M$. We want our new pairing to behave decently on the safe domain. On this domain it will coincide with the following pairing.

$-\langle x, y\rangle_{\rho}:=\langle 0, x, y\rangle$.

By our conventions, we have example:

$$
\langle x, y, z\rangle_{3, \rho}=\langle x,\langle y, z\rangle\rangle_{\rho}=\left\langle 0, x,\langle y, z\rangle_{\rho}\right\rangle_{3}=\langle 0, x, 0, y, z\rangle_{5}
$$

We now define a direct translation $v$ from the language of PAIR $\mathrm{O}_{\mathrm{O}, \mathrm{ns}}^{\mathrm{fun}}$ into the language of $U$.

$-\quad 0_{v}:=0$

$$
-\langle x, y\rangle_{v}:= \begin{cases}\langle 1, w, z\rangle & \begin{array}{l}
\text { if, for some } w, z,\langle 0, w, z\rangle_{\rho}=x \text { and } \\
\langle 1, w, z\rangle=y \text { and } \mathbf{s} w=z
\end{array} \\
\cdots & \\
\langle j+2, \mathbf{z}\rangle & \text { if, for some } \mathbf{z}: D_{0}, \text { we have }\langle j+1, \mathbf{z}\rangle_{\rho}=x \text { and } \\
\cdots & \langle j+2, \mathbf{z}\rangle=y \text { and } P_{j, \mu} \mathbf{z} \\
\langle x, y\rangle_{\rho} & \text { otherwise }\end{cases}
$$

It is easy to see that we defined a functional non-surjective pairing. We really need the functionality both of pairing and of 0 (and consequently of the numerals), even if we do not demand the pairing given by $v$ to be functional. Otherwise, for example, in the first clause, we could have $\langle 0, w, z\rangle_{\rho} \cong x$ and $\langle 0, w, z\rangle_{\rho} \cong x^{\prime}$, where $x \neq x^{\prime}$. Then we would have $\langle x, y\rangle_{v} \cong y$ and $\left\langle x^{\prime}, y\right\rangle_{v} \cong y$, contradicting the properties of pairing. We verify that $D_{0}$ is "safe", i.e., that the complicated $v$ behaves like the simpler $\rho$ on $D_{0}$.

Lemma 2 We have in $U$ : 
1. If $y$ is in $D_{0}^{+}$, then $\langle x, y\rangle_{v}=\langle x, y\rangle_{\rho}=\langle 0, x, y\rangle=\mathbf{s}\langle x, y\rangle$ and, hence, in $D_{0}$.

2. If $y$ is in $D_{0}^{+}$, then $\langle\mathbf{x}, y\rangle_{v}$ is in $D_{0}^{+}$and $\langle\mathbf{x}, y\rangle_{\nu}=\langle\mathbf{x}, y\rangle_{\rho}$.

3. $n_{v}=n_{\rho}=2 n$.

The proof of the lemma is easy. Now consider the first clause of the definition of $v$. Since $z$ is a successor, it is in $D_{0}$. So, we have $\langle 0, w, z\rangle_{v}=\langle 0, w, z\rangle_{\rho}=x$. Similarly, in the clause involving $P_{j, \mu}$, we find that $\langle j+1, \mathbf{z}\rangle_{\nu}=\langle j+1, \mathbf{z}\rangle_{\rho}=x$. Note that we need here that the last of the $\mathbf{z}$ is in $D_{0}$.

Lemma 3 We have in $U: \exists v\langle\langle 0, x, y\rangle, v\rangle_{v}=v \leftrightarrow \mathrm{s} x=y$.

Proof Suppose $\mathrm{s} x=y$. Let $\langle 0, x, y\rangle_{\rho}=u$ and $\langle 1, x, y\rangle=v$. Then, by definition, $\langle u, v\rangle_{v}=v$. Moreover, since $y$ is in $D_{0}, u=\langle 0, x, y\rangle_{\rho}=\langle 0, x, y\rangle_{v}$. Hence $\langle\langle 0, x, y\rangle, v\rangle_{v}=v$.

Conversely, suppose that $\langle\langle 0, x, y\rangle, v\rangle_{v}=v$. So, for some $u,\langle 0, x, y\rangle_{v}=u$ and $\langle u, v\rangle_{v}=v$. We have:

a. If $\langle u, v\rangle_{v}$ was determined by the last clause of the definition, we would have $\langle 0, u, v\rangle=v$, contradicting $(\dagger)$.

b. Suppose $\langle u, v\rangle_{v}$ is determined by the first clause. We have, for some $w, z, \mathbf{s} w=z$ and $\langle 0, w, z\rangle_{\rho}=u$. It follows that $\langle 0, w, z\rangle_{v}=u$, and, hence, $x=w$ and $y=z$. So, $\mathrm{s} x=y$.

c. Suppose $\langle u, v\rangle_{v}$ is determined by the clause involving $P_{j, \mu}$. We have, for some $\mathbf{z}$ in $D_{0}$, that $\langle j+1, \mathbf{z}\rangle_{\rho}=u$. It follows that $\langle j+1, \mathbf{z}\rangle_{v}=u$. This contradicts the fact that $\langle 0, x, y\rangle_{v}=u$.

Lemma 4 In $U:$ for $\mathbf{z}$ in $D_{0}$, we have:

$$
\exists v\langle\langle j+1, \mathbf{z}\rangle, v\rangle_{v}=v \leftrightarrow P_{j, \mu} \mathbf{z}
$$

It follows that, for any a:

$$
\exists \mathbf{z}\left(\bigwedge_{i} \mathrm{~s} a_{i}=z_{i} \wedge \exists v\langle\langle j+1, \mathbf{z}\rangle, v\rangle_{v}=v\right) \leftrightarrow P_{j} \mathbf{a} .
$$

Again it follows that, for any a:

$$
\left(\exists \mathbf{z}\left(\bigwedge_{i}\left(\exists w\left\langle\left\langle 0, a_{i}, z_{i}\right\rangle, w\right\rangle=w\right) \wedge \exists v\langle\langle j+1, \mathbf{z}\rangle, v\rangle=v\right)\right)^{v} \leftrightarrow P_{j} \mathbf{a} .
$$

Proof We prove the first equivalence. Suppose $P_{j, \mu} \mathbf{z}$. Let $\langle j+1, \mathbf{z}\rangle_{\rho}=u$ and let $\langle j+2, \mathbf{z}\rangle=v$. Then, by definition, $\langle u, v\rangle_{v}=v$. Moreover, we have $\langle j+1, \mathbf{z}\rangle_{\rho}=$ $\langle j+1, \mathbf{z}\rangle_{v}$. Hence $\langle\langle j+1, \mathbf{z}\rangle, v\rangle_{v}=v$.

Conversely, suppose that $\langle\langle j+1, \mathbf{z}\rangle, v\rangle_{v}=v$. So, for some $u,\langle j+1, \mathbf{z}\rangle_{v}=u$ and $\langle u, v\rangle_{v}=v$. We have: 
a. If $\langle u, v\rangle_{v}$ was determined by the last clause of the definition, we would have $\langle 0, u, v\rangle=v$, contradicting $(\dagger)$.

b. Suppose $\langle u, v\rangle_{v}$ is determined by the first clause. We have, for some $w, a$, that $\langle 0, w, a\rangle_{\rho}=u$ and $a=\mathrm{s} w$. It follows that $\langle 0, w, a\rangle_{v}=u$. This contradicts the fact that $\langle j+1, \mathbf{z}\rangle_{v}=u$.

c. Suppose $\langle u, v\rangle_{v}$ is determined by the clause involving $P_{k, \mu}$. We have, for some c in $D_{0}$, that $\langle k+1, \mathbf{c}\rangle_{\rho}=u$. It follows that $\langle k+1, \mathbf{c}\rangle_{v}=u$. We also have that $\langle j+1, \mathbf{z}\rangle_{v}=u$. It follows that $j=k$. We may conclude that the lengths of $\mathbf{z}$ and $\mathbf{c}$ are equal and, thus, that $\mathbf{z}=\mathbf{c} .^{14}$

The second equivalence follows immediately from the first, and the third equivalence follows immediately from the second one.

We define a direct translation $\alpha$ from the language of $U$ to the language of PAIR ${ }_{\mathrm{o}, \mathrm{ns}}^{\text {fun }}$. - $P_{j, \alpha} \mathbf{z}: \leftrightarrow \exists \mathbf{z}\left(\bigwedge_{i}\left(\exists w\left\langle\left\langle 0, a_{i}, z_{i}\right\rangle, w\right\rangle=w\right) \wedge \exists v\langle\langle j+1, \mathbf{z}\rangle, v\rangle=v\right)$.

Lemma 4 tells us that $U \vdash\left(P_{j} \mathbf{z}\right)^{\alpha \nu} \leftrightarrow P_{j} \mathbf{z}$. It follows that, for any $U$-formula $B$, we have $U \vdash B \leftrightarrow B^{\alpha \nu}$.

Let $W$ be PAIR fun plus all $\vdash A^{\alpha}$, where $\vdash A$ is an axiom of $U$, plus the axiom $\vdash(\operatorname{pair}(u, v, w))^{v \alpha} \leftrightarrow \operatorname{pair}(u, v, w)$. Clearly, $K:=\langle U, \alpha, W\rangle$ is an interpretation. Moreover we have:

i. $\quad U \vdash\left(\mathrm{PAIR}_{\mathrm{o}, \mathrm{ns}}^{\text {fun }}\right)^{v}$.

ii. Consider any axiom $A$ of $U$. We have $U \vdash A$, and, hence $U \vdash A^{\alpha \nu}$.

iii. We have $U \vdash(\operatorname{pair}(u, v, w))^{\nu} \leftrightarrow(\operatorname{pair}(u, v, w))^{\nu \alpha \nu}$. So, we may conclude: $U \vdash\left(\operatorname{pair}(u, v, w) \leftrightarrow(\operatorname{pair}(u, v, w))^{v \alpha}\right)^{v}$.

So, $L:=\langle W, v, U\rangle$ is an interpretation. Evidently, $K$ is an isomorphism with inverse $L$, and, thus, $U$ is definitionally equivalent to $W$.

\section{The adaptiveness of weak set theory}

In this section we show that WS is adaptive. It is easy to see that, if we had one extra free floating unary predicate $P$, we could adapt the reasoning of Example 6 to do the trick. All the work for our main result is to simulate the presence of such a $P$. To implement this, we will build a set that is definable modulo extensional identity in a suitable extension of WS such that certain elements of this set will do the work of $P$.

\subsection{Restriction}

In this subsection, we provide a method of restricting our sets to definable classes of sets, plus the universal set: all sets outside the given class will be blown up to the universal set. (We opt for blowing up rather than shrinking these sets to the empty one, because this gives us better absoluteness properties for our restriction construction).

\footnotetext{
$\overline{14}$ Remember that, in our coding of sequences, every representation of a sequence is also a representation of a sequence of any smaller non-zero length. Thus, to identify sequences, one needs contextual information about their intended length.
} 
We work in WS. Suppose that $\mathcal{X}$ is a definable class such that, provably, $\mathcal{X}$ contains all empty sets and is closed under $\mathrm{S}_{y}$, i.e.:

$$
\forall x \in \mathcal{X} \forall y, z\left(\mathrm{~S}_{y} x \cong z \rightarrow z \in \mathcal{X}\right)
$$

We define the direct translation $\rho \mathcal{X}$ of the language of WS in itself as follows.

$-\quad x \in_{\rho_{\mathcal{X}}} y: \leftrightarrow(x \in y \vee y \notin \mathcal{X})$.

As is easily seen $\rho \mathcal{X}$ supports an interpretation $R_{\mathcal{X}}:$ WS $\triangleright$ WS. We define:

$-\left\{x_{0}, \ldots, x_{n-1}\right\} \cong y: \leftrightarrow \forall z\left(z \in y \leftrightarrow \bigvee_{j<n} z=x_{j}\right)$

We claim that $\left\{x_{0}, \ldots, x_{n-1}\right\}$ is absolute w.r.t. $\rho:=\rho \mathcal{X}$ in the sense that, provably, $\left\{x_{0}, \ldots, x_{n-1}\right\}_{\rho}$ is equal to $\left\{x_{0}, \ldots, x_{n-1}\right\}$, or, i.o.w.,

$$
\forall y\left(\forall z\left(z \in \in_{\rho} y \leftrightarrow \bigvee_{j<n} z=x_{j}\right) \leftrightarrow \forall z\left(z \in y \leftrightarrow \bigvee_{j<n} z=x_{j}\right)\right)
$$

First suppose that $\forall z\left(z \in y \leftrightarrow \bigvee_{j<n} z=x_{j}\right)$. In this case, $\mathrm{S}_{x_{0}} \ldots \mathrm{S}_{x_{n-1}} \emptyset \cong y$, and, hence, $y \in \mathcal{X}$. So, by the definition of $\rho$, we are immediately done.

Next suppose $\forall z\left(z \in \rho \leftrightarrow \bigvee_{j<n} z=x_{j}\right)$. Suppose also that $y \notin \mathcal{X}$. It follows that $\forall z \bigvee_{j<n} z=x_{j}$. We show that this is impossible. We define:

$-\lceil 0\rceil:=\emptyset,\lceil k+1\rceil:=\mathrm{S}_{\lceil k\rceil}\lceil k\rceil$.

It is easy to show that each $\lceil k\rceil$ contains precisely $k$ elements and, thus, that there are more than $n$ elements in our domain. A contradiction. We may conclude that $y \in \mathcal{X}$. So, by the definition of $\rho$, we are immediately done.

We can easily show that absoluteness w.r.t. direct translations is preserved under composition of multifunctions, i.e, if the $k$-ary $F$ and $G_{0}, \ldots, G_{k-1}$ are absolute, then so is $\lambda \mathbf{y} \cdot F\left(G_{0}(\mathbf{y}), \ldots, G_{k-1}(\mathbf{y})\right)$. It follows that, $\langle x, y\rangle$ is absolute w.r.t. $\rho \mathcal{X}$. Also the $\lceil n\rceil$ are absolute.

\subsection{Adding intersections}

In this subsection we construct a class of sets that is closed under intersection.

We work in WS. Let $x \cap y \cong z: \leftrightarrow \forall u(u \in z \leftrightarrow(u \in x \wedge u \in y))$. We define the class $\mathcal{X}_{0}:=\{x \mid \forall y \quad y \cap x \downarrow\}$. We show that $\mathcal{X}_{0}$ is closed under empty sets, addition of an element and under intersection. Closure under empty sets is trivial.

Consider $x$ in $\mathcal{X}_{0}$ and consider any $z$ and $y$. Suppose that $\mathrm{S}_{z} x \cong u$ and $y \cap x \cong v$. In case $z \in y$, we can take $y \cap u: \cong w$, for any $w$ with $\boldsymbol{S}_{z} v \cong w$. In case $z \notin y$, we can take $y \cap u: \cong v$.

Consider any $x_{0}$ and $x_{1}$ in $\mathcal{X}_{0}$. Suppose $x_{0} \cap x_{1} \cong u$. We have to show that, for any $y, y \cap u \downarrow$. Clearly, for some $v,\left(\left(y \cap x_{0}\right) \cap x_{1}\right) \cong v$. So, we can take $y \cap u: \cong v$.

It is easily seen that $\rho \mathcal{X}_{0}$ supports $R^{0}: \mathrm{WS} \triangleright_{\text {dir }}$ (WS + intersection), where intersection is the axiom that intersection is a total multifunction. 


\subsection{The Russell construction}

We need to have sets from which at least some items of a specific 'form' are lacking. To implement this, we can use the familiar argument leading to the Russell paradox.

We work in WS + intersection. Let $F$ be any definable unary multifunction that is provably total and injective modulo extensional equality in the following sense: if $F x_{0} \cong y$ and $F x_{1} \cong y$, then $x_{0}=$ ext $x_{1}$. We define:

- $\mathrm{R}_{x}^{F} \cong y: \leftrightarrow \forall z(z \in y \leftrightarrow(z \in x \wedge \exists u(F u \cong z \wedge z \notin u)))$.

We show that:

$$
\forall r, f\left(\left(\mathrm{R}_{x}^{F} \cong r \wedge F r \cong f\right) \rightarrow f \notin x\right)
$$

Let $\mathrm{R}_{x}^{F} \cong r$ and $F r \cong f$. Suppose $f \in r$. Then, $f \in x$ and, for some $u, F u \cong f$ and $f \notin u$. By the injectivity of $F$ modulo extensional equality, we have $u={ }_{\text {ext }} r$. Hence, $f \notin r$, a contradiction. So, we may conclude that $f \notin r$. Suppose $f \in x$. Then, we have: $f \in x, F r \cong f, f \notin r$. So, $f \in r$. A contradiction. We may conclude that $f \notin x$.

Let $\mathcal{X}_{1}$ be the class of all $x$ such that $\mathrm{R}_{x}^{F} \downarrow$. Clearly, $\mathcal{X}_{1}$ is closed under empty sets and addition of an element. Moreover, since we have intersections, $\mathcal{X}_{1}$ is downwards closed under $\subseteq$. Note that it follows that, for $x \in \mathcal{X}_{1}$, and $\mathrm{R}_{x}^{F} \cong y$, we have $y \in \mathcal{X}_{1}$.

Suppose $F$ is absolute w.r.t. $\rho_{1}:=\rho \mathcal{X}_{1}$. We write $\mathrm{V} \cong x$ for: $\forall u u \in x$. We claim that $\rho_{1}$ supports $R^{1}$ : (WS + intersection) $\triangleright_{\text {dir }} W_{F}$, where:

$$
W_{F}:=\mathrm{WS}+\text { intersection }+\forall x(\mathrm{~V} \cong x \vee \exists y \forall z(F y \cong z \rightarrow z \notin x))
$$

It is easy to see that, in WS + intersection, we have closure under empty sets, addition of elements and intersection in the theory interpreted via $\rho_{1}$. It follows that $F$ is total and injective modulo extensional equality in the interpreted theory.

We prove the last principle. Consider any $x$. We first suppose $x \notin \mathcal{X}_{1}$. Then, $\mathrm{V}_{\rho_{1}} \cong x$. Next suppose $x \in \mathcal{X}_{1}$. Pick $y$ such that $\mathrm{R}_{x}^{F} \cong y$. Since $x \in \mathcal{X}_{1}$, we have $y \in \mathcal{X}_{1}$. Suppose that $F_{\rho_{1}} y \cong z$. It follows that $F y \cong z$. So, by Equation 1 , we have $z \notin x$, and, thus, $z \notin \rho_{1} x$. $^{15}$

\subsection{The main construction}

In this subsection, we provide the main ingredient of our argument.

Par abus de langage, we write 0 for $\lceil 0\rceil$ and 1 for $\lceil 1\rceil$. We define an auxiliary direct translation $\alpha$ as follows:

$-\quad x \in_{\alpha} y: \leftrightarrow \forall u(\langle 0, u\rangle \cong y \rightarrow x \in u)$.

Note that if $y$ is not of the form $\langle 0, u\rangle$, then $y$ represents the universal set $\mathrm{V}_{\alpha}$. Clearly, we have an interpretation $K_{\alpha}$ based on $\alpha$ such that $K_{\alpha}$ :WS $\triangleright$ WS.

$\overline{15}$ Note that $\mathbf{R}_{x}^{F}$ need not be absolute w.r.t. $\rho_{1}$. 
We may show by an argument, analogous to the proof that $\left\{x_{0}, \ldots, x_{n-1}\right\}$ is absolute w.r.t. $\rho \mathcal{X}$, that $\left\{x_{0}, \ldots, x_{n-1}\right\}_{\alpha}=\left\langle 0,\left\{x_{0}, \ldots, x_{n-1}\right\}\right\rangle$. We define $\mathrm{F} x:=\langle 0, x\rangle_{\alpha}$. It follows that:

$$
\mathrm{F} x=\langle 0,\{\langle 0,\{\langle 0,0\rangle\}\rangle,\langle 0,\{\langle 0,0\rangle, x\}\rangle\}\rangle .
$$

It is easy to see that $\mathrm{F}$ is total and injective (and, thus, a fortiori, injective w.r.t. extensional equality) because $K_{\alpha}$ is a direct interpretation of WS. By its definition $\mathrm{F}$ is absolute w.r.t. any $\rho \mathcal{X}$. Let $W$ be the "Russell theory" $W_{\mathrm{F}}$ (as introduced in the previous subsection).

Suppose $U \triangleright_{\text {dir }}$ WS. It follows that we have a direct interpretation $N: U \triangleright_{\operatorname{dir}} W$. Let $v$ be the translation associated to $N$. We work in $U$. We suppress the subscript and superscript $v$ connected to set theoretical notions as given by $N$.

Suppose the predicate symbols of $U$ are $P_{0}, \ldots, P_{n-1}$. We define a direct translation $\beta$ of the language of WS as follows.

$-\square:=[]_{0}:=\emptyset,[\mathbf{x}, y]_{n+1}:=\left\langle[\mathbf{x}]_{n}, y\right\rangle$. Par abus de langage we will suppress the subscripts in our sequence notation.

$-\mathrm{C}_{0}(x): \leftrightarrow \exists v\langle 0, v\rangle_{\alpha} \cong x$,

$-\mathrm{C}_{i+1}(x): \leftrightarrow \exists \mathbf{y}\left(\langle\lceil i+1\rceil,[\mathbf{y}]\rangle_{\alpha} \cong x \wedge P_{i} \mathbf{y}\right)$.

$-y \in_{\beta} x: \leftrightarrow \exists u(\langle 0, u\rangle \cong x \wedge y \in u)$

$$
\begin{aligned}
& \vee \exists u\left(\langle 1, u\rangle \cong x \wedge\left(y \in u \vee \bigvee_{j} \mathrm{C}_{j}(y)\right)\right) \\
& \vee \forall u \neg(\langle 0, u\rangle \cong x \vee\langle 1, u\rangle \cong x)
\end{aligned}
$$

Clearly, for any $\langle 0, \emptyset\rangle \cong u$, we have $\emptyset_{\beta} \cong u$. Consider any $x$. In case, for no $u$, we have $\langle 0, u\rangle \cong x$ or $\langle 1, u\rangle \cong x$, we find $\mathrm{S}_{y, \beta} x \cong x$. If $\langle j, u\rangle \cong x$, for $j=0$, 1, we have, for any $v$ with $\left\langle j, \mathrm{~S}_{y} u\right\rangle \cong v$, that $\mathrm{S}_{y, \beta} x \cong v$. Hence, $\beta$ yields an interpretation of WS.

We say that $A \mathbf{y}$ is $\alpha, \beta$-absolute, if, provably in $U, \forall \mathbf{y}\left(A^{\alpha} \mathbf{y} \leftrightarrow A^{\beta} \mathbf{y}\right) . G$ is $\alpha, \beta$-absolute iff $G \mathbf{y} \cong z$ is $\alpha, \beta$-absolute. We show that $\left\{x_{0}, \ldots, x_{n-1}\right\}$ is $\alpha, \beta$-absolute. We have to show:

$$
\forall z\left(z \in_{\alpha} y \leftrightarrow \bigvee_{j<n} z=x_{j}\right) \leftrightarrow \forall z\left(z \in_{\beta} y \leftrightarrow \bigvee_{j<n} z=x_{j}\right) .
$$

Suppose that $\forall z\left(z \in_{\alpha} y \leftrightarrow \bigvee_{j<n} z=x_{j}\right)$. In case we do not have $\langle 0, v\rangle \cong y$, for any $v$, we get $\forall z \bigvee_{j} z=x_{j}$. Quod impossible. So, $\langle 0, v\rangle \cong y$, for some $v$. It follows that $\forall z\left(z \in_{\alpha} y \leftrightarrow z \in \beta \quad y\right)$, so we are done.

Suppose that $\forall z\left(z \in \beta \quad y \leftrightarrow \bigvee_{j<n} z=x_{j}\right)$. Suppose $\langle 1, v\rangle \cong y$, for some $v$. We have, for some $\mathbf{w},\langle 0,\lceil 0\rceil\rangle_{\alpha} \cong w_{0} \in_{\beta} y, \ldots,\langle 0,\lceil n\rceil\rangle_{\alpha} \cong w_{n} \in_{\beta} y$. Since the $\langle 0,\lceil n\rceil\rangle_{\alpha}$ are pairwise disjoint, we have a contradiction. So, for no $v,\langle 1, v\rangle \cong y$. In the other cases, we are easily done.

It is easily seen that the $\alpha, \beta$-absolute multifunctions are closed under composition. Thus, it follows that $\langle x, y\rangle_{\alpha}=\langle x, y\rangle_{\beta},\lceil n\rceil_{\alpha}=\lceil n\rceil_{\beta}$, etc.

We define the following property:

$-Q_{0}(x): \leftrightarrow \forall y, z(\langle 0, y\rangle \cong z \rightarrow z \in x)$. 
Suppose $Q_{0}^{\beta}(x)$. We show that, if $\langle 0, u\rangle \cong x$, then $\mathrm{V}_{\beta} \cong x$. Suppose that $\langle 0, u\rangle \cong x$. It follows that, for all $y$ and $z$ with $\langle 0, y\rangle_{\beta} \cong z$, we have $z \in u$. Hence, by the $\alpha$, $\beta$-absoluteness of $\langle 0, u\rangle$, we find that for all $y$ and $z$ with $\langle 0, y\rangle_{\alpha} \cong z$, we have $z \in u$. By the Russellian principle of $W$, we find $\mathrm{V} \cong u$ and hence $\mathrm{V}_{\beta} \cong x$.

Note that, if, for no $u,\langle 0, u\rangle \cong x$ or $\langle 1, u\rangle \cong x$, then again $\mathrm{V}_{\beta} \cong x$. So, if $Q_{0}^{\beta}(x)$ and, for no $u,\langle 1, u\rangle \cong x$, then $\mathrm{V}_{\beta} \cong x$.

Now suppose that $\langle 1, u\rangle \cong x$. The $\mathrm{C}_{0}$-disjunct in the definition of $\in_{\beta}$ tells us that, for any $y$ and $z$ with $\langle 0, y\rangle_{\alpha} \cong z$, we have $z \in_{\beta} x$. By the $\alpha, \beta$-absoluteness of $\langle 0, y\rangle$, we find that $Q_{0}^{\beta}(x)$.

Let $\langle 1, \emptyset\rangle \cong x^{\star}$. It is now easily seen that $Q_{0}^{\beta}\left(x^{\star}\right)$, and that, for all $x$ with $Q_{0}^{\beta}(x)$, we have $x^{\star} \subseteq{ }^{\beta} x$. Let:

$-Q(x): \leftrightarrow Q_{0}(x) \wedge \forall y\left(Q_{0}(y) \rightarrow x \subseteq y\right)$.

We have shown that $Q^{\beta}\left(x^{\star}\right)$. Note that, trivially, whenever $Q^{\beta}(v)$ and $Q^{\beta}(w)$, then $v={ }_{\text {ext }}^{\beta} w$.

Until now we have worked in $U$. We have defined a translation $\gamma:=\beta \circ v$ of the language of WS into the language of $U$ with various desirable properties. We are now ready to define the translation $\tau$ of the language of $U$ into the language of WS. This translation is intended to be "inverse" to $\gamma$. We define:

$-\quad P_{j, \tau} \mathbf{y}: \leftrightarrow \exists x(Q(x) \wedge \exists w(\langle\lceil j+1\rceil,[\mathbf{y}]\rangle \cong w \wedge w \in x))$.

$-V:=\mathrm{WS}+\left\{A^{\tau} \mid A \in \mathrm{ax}_{U}\right\}+\forall x, y\left(x \in y \leftrightarrow(x \in y)^{\gamma \tau}\right)$.

Trivially, $M:=\langle U, \tau, V\rangle$ is an interpretation. We verify that $K:=\langle V, \gamma, U\rangle$ is an interpretation. We verified that $U \vdash \mathrm{WS}^{\gamma}$.

Lemma 5 We have: $U \vdash P_{j} \mathbf{y} \leftrightarrow P_{j, \tau}^{\gamma} \mathbf{y}$.

Proof We have $P_{j, \tau}^{\gamma} \mathbf{y}$ iff $\exists x\left(Q^{\gamma}(x) \wedge \exists w\left(\langle\lceil j+1\rceil,[\mathbf{y}]\rangle_{\gamma} \cong w \wedge w \in_{\gamma} x\right)\right)$. We have shown that $Q^{\gamma}$ is satisfied by an $x^{\star}$ that is unique modulo $\gamma$-extensional equality. This $x^{\star}$ can be taken such that $\langle 1, \emptyset\rangle \cong x^{\star}$. Thus, we have: $P_{j, \tau}^{\gamma} \mathbf{y}$ iff $\exists w\left(\langle\lceil j+1\rceil,[\mathbf{y}]\rangle_{\gamma} \cong\right.$ $\left.w \wedge w \in_{\gamma} x^{\star}\right)$.

We have $w \in_{\gamma} x^{\star}$ iff, $w$ is of the form $\langle 0, v\rangle_{\alpha \nu}$, or, for some $\mathbf{v}$,

$$
\langle\lceil i+1\rceil,[\mathbf{v}]\rangle_{\alpha \nu} \cong w \text { and } P_{i} \mathbf{v}
$$

By $\alpha, \beta$-absoluteness, $w \in_{\gamma} \quad x^{\star}$ iff, $w$ is of the form $\langle 0, v\rangle_{\gamma}$, or, for some $\mathbf{v}$, $\langle\lceil i+1\rceil,[\mathbf{v}]\rangle_{\gamma} \cong w$ and $P_{i} \mathbf{v}$. Thus, we have, by the properties of pairing, $\left(\langle\lceil j+1\rceil,[\mathbf{y}]\rangle_{\gamma} \cong w \wedge w \in_{\gamma} x^{\star}\right)$ iff $P_{j} \mathbf{y}$.

By the lemma, we find that, for any axiom $A$ of $U, U \vdash A^{\tau \gamma}$. Moreover, we have:

$$
U \vdash(x \in y)^{\gamma} \leftrightarrow(x \in y)^{\gamma \tau \gamma} .
$$

Hence, $U \vdash\left(x \in y \leftrightarrow(x \in y)^{\gamma \tau}\right)^{\gamma}$. It follows that $K$ is an interpretation.

It is now immediate that $K$ and $M$ witness the fact that $U$ and $V$ are isomorphic. 
Remark 4 Alternatively, we could set up the argument as follows. We define $V$ to be the theory given by $\left\{A \in\right.$ sentws $\left._{\text {w }} \mid U \vdash A^{\gamma}\right\}$. We can give a p-time decidable axiomatization of this theory using a version of Craig's trick. Now it is clear that $M:=\langle U, \tau, V\rangle$ and $K:=\langle V, \gamma, U\rangle$ are interpretations and that $K$ is a split epi. Moreover, $K$ is faithful (by construction) and, hence, mono. (see [21]) Ergo, $K$ is an isomorphism.

Acknowledgments I am grateful to Lev Beklemishev and Pavel Pudlák for their advice on matters of definition. I am grateful to Rosalie Iemhoff and Ali Enayat for stimulating conversations. I thank Rosalie for her comments on the manuscript. Finally, I thank the anonymous referees for their corrections and comments.

Open Access This article is distributed under the terms of the Creative Commons Attribution Noncommercial License which permits any noncommercial use, distribution, and reproduction in any medium, provided the original author(s) and source are credited.

\section{Appendix A: Interpretations with parameters}

In this appendix we treat interpretations with parameters. A translation with parameters is of the form $\tau=\langle\mathbf{w}, \delta, F\rangle$, where the $\mathbf{w}$ are the parameters. ${ }^{16}$ Such a translation is defined as the obvious adaptation of the unparametrized version. For e.g., we take $P_{\tau} \mathbf{v}$ to be a formula $B \mathbf{w v}$ with free variables among $\mathbf{w}, \mathbf{v}$. Here, the $\mathbf{w}$ are supposed to be disjoint from the $\mathbf{v}$. The domain $\delta$ is treated similarly. An interpretation with parameters is a quadruple $\langle U, \tau, V, A\rangle$. Here $\tau=\langle\mathbf{w}, \delta, F\rangle$ is a translation from the language of $U$ to the language of $V$. The formula $A$ is a $V$-formula with at most $\mathbf{w}$ free. We demand:

$-\quad V \vdash \exists \mathbf{w} A$,

- for all $U$-sentences $B$, if $U \vdash B$, then $V \vdash \forall \mathbf{w}\left(A \rightarrow B^{\tau}\right)$.

Note that the second clause takes care automatically of non-emptiness of the domain and the laws of identity. We also write $\langle K, A\rangle$ for $\langle U, \tau, V, A\rangle$. If we want to stress the presence of the parameters, we write $K \mathbf{w}$, etc.

The identity interpretation $\mathrm{id}_{T}: T \rightarrow T$ has parameters $\square$ and is of the form $\left\langle\operatorname{id}_{\Sigma}, \top\right\rangle$, where $\Sigma$ is the signature of $T$. Suppose $\langle K, A\rangle: U \rightarrow V$ and $\langle M, B\rangle$ : $V \rightarrow W$. We wish to define $\langle N, C\rangle:=(\langle M, B\rangle \circ\langle K, A\rangle): U \rightarrow W$. Suppose $\mathbf{w}$ is the parameter sequence of $\langle M, B\rangle$ and $\mathbf{z}$ is the parameter sequence of $\langle K, A\rangle$. If necessary, we make these sequences disjoint (with the obvious adaptations of the translations). The parameter sequence of the composition is wz. The composition of the underlying translations is closely analogous to the case without parameters. The parameter formulas transform as follows:

$-C \mathbf{w z}: \leftrightarrow B \mathbf{w} \wedge \mathbf{z}: \delta_{M \mathbf{w}} \wedge A^{M \mathbf{w}} \mathbf{z}$.

The category of interpretations with parameters is a terra incognita. So, the sketchy remarks that follow are only tentative. To form a category INT ${ }^{\text {par }}$ of interpretations with parameters, we have to say when two interpretations are equal. Some experimentation

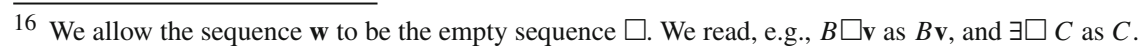


suggests the following definition. Suppose $\langle K, A\rangle: U \rightarrow V$ and $\langle M, B\rangle: U \rightarrow V$. We define:

$$
\begin{gathered}
-\langle K, A\rangle \preceq\langle M, B\rangle: \leftrightarrow \forall \mathbf{w}: A \exists \mathbf{z}: B\left(\forall v\left(\delta_{K \mathbf{w}} v \leftrightarrow \delta_{M \mathbf{z}} v\right)\right. \\
\left.\wedge \forall \mathbf{v}: \delta_{K \mathbf{w}}\left(P_{K \mathbf{w}} \mathbf{v} \leftrightarrow P_{M \mathbf{z}} \mathbf{v}\right) \wedge \ldots\right) .
\end{gathered}
$$

It is easy to see that $\preceq$ defines a preorder on interpretations. We take the induced equivalence relation of $\preceq$ as our notion of equality of interpretations.

The fact that this notion of equality is a natural choice can be seen as follows. The interpretation $\langle K, A\rangle$ can be viewed as a uniform construction of an $A$-indexed set of internal models of $U$ inside a model of $V$. The obtaining of the relation $\langle K, A\rangle \preceq$ $\langle M, B\rangle$ tells us that the $\langle K, A\rangle$-models are a subset of the $\langle M, B\rangle$-models.

We can now show that we defined a category INT ${ }^{\text {par }}$. If we enrich this category with the relations $\preceq$ between morphisms, we obtain a 2-category.

We define $\langle K, A\rangle^{-1}[V]$ to be the theory, suitably axiomatized, with theorems $\left\{B \in \operatorname{sent}_{U} \mid V \vdash \forall \mathbf{w}: A B^{K \mathbf{w}}\right\}$. We obviously have:

Theorem 10 If $\langle K, A\rangle \preceq\langle M, B\rangle$, then $\langle M, B\rangle^{-1}[V] \subseteq\langle K, A\rangle^{-1}[V]$. It follows that, if $\langle K, A\rangle \preceq\langle M, B\rangle$ and if $\langle K, A\rangle$ is faithful, then so is $\langle M, B\rangle$.

We remind the reader of Tarski's way of treating interpretations with parameters. (see [14]) A theory $W$ is called an inessential extension of a theory $V$ if, for some formula $A \mathbf{w}$, we have $V \vdash \exists \mathbf{w} A \mathbf{w}$, and, and for some fresh constants corresponding to the $\mathbf{w}$, we have $W=V+A \mathbf{c}$, where the signature of $W$ is the signature of $V$ expanded by c. Tarski defines an interpretation with parameters $\mathbf{w}$ as an ordinary interpretation in an inessential extension with fresh constants $\mathbf{c}$ matching $\mathbf{w}$.

Tarski's definition is justified, from our point of view, by the following immediate insights:

$$
\langle K, A\rangle: U \rightarrow V \Leftrightarrow K \mathbf{c}: U \rightarrow(V+A \mathbf{c}) .
$$

Moreover, we have a splitting of $\langle K, A\rangle: U \rightarrow V$ :

$$
U \stackrel{\langle K \mathbf{c}, T\rangle}{\longrightarrow}(V+A \mathbf{c}) \stackrel{\left\langle\gamma_{A}, A\right\rangle}{\longrightarrow} V .
$$

Here $\gamma_{A}$, is based on the direct translation that sends $C_{i} v_{0}$, the unary predicate representing the constant $c_{i}$, to $w_{i}=v_{0}$. It is easy to see that $\left\langle\gamma_{A}, A\right\rangle$ is a retraction (or: split epimorphism) in INT ${ }^{\text {par }}$, with as corresponding coretraction (or split monomorphism) the expansion $\langle\varepsilon, \top\rangle: V \rightarrow(V+A \mathbf{c})$.

Note that $\left\langle\gamma_{A}^{+}, A\right\rangle:=\langle\varepsilon, \top\rangle \circ\left\langle\gamma_{A}, A\right\rangle$ is not the identity $\left\langle\mathrm{id}_{V+A \mathbf{c}}, \top\right\rangle$, since in going back and forth we lose our "specific choice" of c. However, we do have: $\left\langle\operatorname{id}_{V+A c}, T\right\rangle \preceq$ $\left\langle\gamma_{A}^{+}, A\right\rangle$, which shows that $\left\langle\gamma_{A}^{+}, A\right\rangle$ is faithful. ${ }^{17}$

Question 7 We could plausibly call an inessential extension a Henkin extension, since it embodies a step in the construction of a Henkin theory from a given theory. This

\footnotetext{
17 Thus, INT ${ }^{\text {par }}$ is not equivalent to the result of identifying over INT a theory and all its inessential extensions, if such an idea makes sense at all.
} 
suggests that we could have a more encompassing notion of interpretation, if we replace Henkin extension by Skolem extension, i.e., extension with a finite set of Skolem functions. It would be interesting to see whether this gives a good notion of interpretation.

\section{Appendix B: Historical notes on sequentiality}

The notion of sequential theory was introduced by Pavel Pudlák [9] in his paper. Pudlák uses his notion for the study of the degrees of local multidimensional parametric interpretability. He proves that sequential theories are prime in this degree structure. In [10], sequential theories provide the right level of generality for theorems about consistency statements.

Remark 5 Pudlák's definition is not precisely the same as ours.

1. He works in [9] with axiom sets of arbitrary complexity and possibly infinite signatures.

2. He uses, as container theory, a theory in the style of our definition of PSEQ, only his theory is lighter. In stead of an embedded theory of arithmetic, he uses a theory of linear order in which each element has a successor. Moreover, he has much more modest demands on the theory of sequences. These differences are inessential modulo mutual direct interpretability.

3. Pudlák does not stipulate an initial element for his ordering. This initial element has to be provided via a parametric interpretation. ${ }^{18}$

4. Pudlák demands that identity on the linear ordering of his container theory is absolute. This corresponds, modulo mutual direct interpretability, with the demand that E of our container theory PSEQ $(T)$ is identity. ${ }^{19}$

The demand that the identity of the embedded linear order (or, in our context, arithmetical theory) is absolute is somewhat arbitrary. The elements of the order (numbers) are part of the implementation of the containers. The precise individuation of the containers does not matter: they just have to do their job. After all, for the same reason, we do not demand that our sequence coding is functional from containees to containers.

Clearly, Pudlák's notion (assuming that we restrict ourselves to enumerable theories of finite signature) is contained in our notion of parametric sequential theory.

Since all the known theorems work for parametric sequentiality in our sense, this notion seems to be a good choice for the notion of sequentiality tout court. ${ }^{20}$

We opted for keeping the designation "sequential" for our weaker notion, just because "unparametric sequentiality" and "non-parametric sequentiality" sound a bit awkward. The result of Sect. 6, in terms of parametric sequentiality, becomes: a theory is parametrically sequential iff it has an inessential extension that is (non-parametrically) definitionally equivalent to an extension of WS.

\footnotetext{
18 One can easily provide a model of Pudlák's container theory that has an automorphism with no fixed points in the linear ordering. Thus, the use of a parametric interpretation is essential.

19 Fact 1 tells us that $\mathrm{Q}$ cannot be interpreted with absolute identity in WS.

20 As Pavel Pudlák pointed out in a personal communication, one could consider generalizing the notion using the idea of direct multidimensional interpretability. See also Remark 1.
} 
At the end of [9], Pudlák introduces the notion of WS-theory in analogy of Vaught's notion of VS-theory. In the paper [7], WS-theories are called weak set theories. I do not think the name "weak set theory" is a happy choice. For e.g., ZFC plus some large cardinal axioms would be a weak set theory, under this terminology.

The notion of sequential theory was independently invented by Friedman who called it adequate theory. See Smoryński's survey [12]. ${ }^{21}$ Friedman uses the notion to provide the Friedman characterization of interpretability among finitely axiomatized sequential theories. (See also [16] and [17]) Moreover, he shows that ordinary interpretability and faithful interpretability among finitely axiomatized sequential theories coincide. (See also [18] and [20])

The most important ingredient of the direct interpretability of PSEQ(Q) in WS is the ordinary interpretability of $Q$ in WS. Here is the brief history of this result.

1. In [13], Wanda Szmielew and Alfred Tarski announce the interpretability of $Q$ in WS plus extensionality (see also [14], p 34).

2. A proof of the Szmielew-Tarski result is given by George Collins and Joseph Halpern in [1].

3. Franco Montagna and Antonella Mancini [6], give an improvement of the Szmielew-Tarski result. They prove that $Q$ can be interpreted in an extension of WS in which we stipulate the functionality of empty set and adjoining of singletons.

4. In appendix III of [7], Jan Mycielski, Pavel Pudlák and Alan Stern provide the ingredients of the interpretation of $Q$ in WS.

In a forthcoming paper we will provide another proof of the interpretability of $Q$ in WS.

A very nice presentation of the converse interpretability of (an extension of) WS plus extensionality in $Q$, is given in [8]. This is an interpretation with absolute identity. We show in Example 3 that such an interpretation cannot be direct, and that, hence, $Q$ is not sequential.

For further work concerning sequential theories, see, e.g., [4,5,7,10,12,18-20].

\section{Appendix C: List of questions}

1. We could plausibly call an inessential extension a Henkin extension, since it embodies a step in the construction of a Henkin theory from a given theory. This suggests that we could have a more encompassing notion of interpretation, if we replace Henkin extension by Skolem extension. It would be interesting to see whether this gives a good notion of interpretation. This is Question 7.

2. Is there a sequential arithmetical theory that is minimal w.r.t. direct interpretability? This is Question 1.

3. It is well known that every sequential theory is mutually locally interpretable with an arithmetical theory. Is it also true that every sequential theory is mutually interpretable with an arithmetical theory? This is Question 2.

\footnotetext{
${ }^{21}$ An important difference is that in the definition, as given by Smoryński, Elementary Arithmetic EA (aka $I \Delta_{0}+\mathrm{EXP}$ ) is stipulated to be interpretable in adequate theories. This demand is evidently much too strong.
} 
4. Is adaptiveness definable in terms of INT? This is Question 3.

5. Consider the degree structures DEG ${ }^{\text {dir }}$ of direct interpretability and DEG ${ }^{\text {epi }}$ of epimorphic interpretability. Does the embedding functor from DEG ${ }^{\mathrm{epi}}$ into DEG ${ }^{\text {dir }}$ have a left adjoint? (The elements in the image of such a functor would be precisely the adaptive theories.) If the answer is no, can we find suitable restrictions of the degree structures for which the answer is yes? This is Question 4.

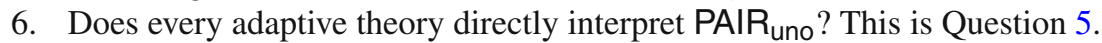

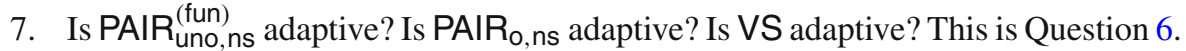

\section{References}

1. Collins, G.E., Halpern, J.D.: On the interpretability of arithmetic in set theory. Notre Dame J. Form. Log. 11, 477-483 (1970)

2. Cégielski, P., Richard, D.: Decidability of the natural integers equipped with the Cantor pairing function and successor. Theor. Comput. Sci. 257(1-2), 51-77 (2001)

3. Ferrante, J., Rackoff, C.W.: The computational complexity of logical theories, vol. 718 of Lecture Notes in Mathematics. Springer, Berlin (1979)

4. Hájek, P., Pudlák, P.: Metamathematics of first-order arithmetic. Perspectives in mathematical logic. Springer, Berlin (1991)

5. Joosten, J.J., Visser, A.: The interpretability logic of all reasonable arithmetical theories. Erkenntnis 53(1-2), 3-26 (2000)

6. Montagna, F., Mancini, A.: A minimal predicative set theory. Notre Dame J. Form. Log. 35, 186-203 (1994)

7. Mycielski, J., Pudlák, P., Stern, A.S.: A lattice of chapters of mathematics (interpretations between theorems), vol. 426 of Memoirs of the American Mathematical Society. AMS, Providence (1990)

8. Nelson, E.: Predicative arithmetic. Princeton University Press, Princeton (1986)

9. Pudlák, P.: Some prime elements in the lattice of interpretability types. Trans. Am. Math. Soc. 280, 255-275 (1983)

10. Pudlák, P.: Cuts, consistency statements and interpretations. J Symb. Log. 50, 423-441 (1985)

11. Quine, W.V.: Reduction to a dyadic predicate. J. Symb. Log. 19(3), 180-182 (1954)

12. Smoryński, C.: Nonstandard models and related developments. In: Harrington, L.A., Morley, M.D., Scedrov, A., Simpson, S.G. (eds.) Harvey Friedman's Research on the foundations of mathematics, pp. 179-229. North Holland, Amsterdam (1985)

13. Szmielew, W., Tarski, A.: Mutual interpretability of some essentially undecidable theories. In: Proceedings of the International Congress of Mathematicians, vol. 1, p. 734. Cambridge (1950)

14. Tarski, A., Mostowski, A., Robinson, R.M.: Undecidable theories. North-Holland, Amsterdam (1953)

15. Vaught, R.A.: Axiomatizability by a schema. J. Symb. Log. 32(4), 473-479 (1967)

16. Visser, A.: Interpretability logic. In: Petkov, P.P. (ed.) Mathematical logic, pp. 175-209. Plenum Press, New York (1990)

17. Visser, A.: An inside view of exp. J. Symb. Log. 57, 131-165 (1992)

18. Visser, A.: The unprovability of small inconsistency. Arch. Math. Log. 32, 275-298 (1993)

19. Visser, A.: An Overview of Interpretability Logic. In: Kracht, M., de Rijke, M., Wansing, H., Zakharyaschev, M. (eds.) Advances in modal logic, vol. 1, issue no. 87, CSLI Lecture Notes, pp. 307-359. Center for the Study of Language and Information, Stanford (1998)

20. Visser, A.: Faith \& Falsity: a study of faithful interpretations and false $\Sigma_{1}^{0}$-sentences. Ann. Pure Appl. Log. 131, 103-131 (2005)

21. Visser, A.: Categories of theories and interpretations. In: Enayat, A., Kalantari, I., Mojtaba, M. (eds.) Logic in Tehran. Proceedings of the Workshop and Conference on Logic, Algebra and Arithmetic, held October 18-22, 2003, vol. 26 of Lecture Notes in Logic, pp. 284-341. ASL, A.K. Peters, Ltd, Wellesley, Mass (2006)

22. Visser, A.: Prolegomena to the categorical study of interpretations. Logic Group Preprint Series 249, Department of Philosophy, Utrecht University, Heidelberglaan 8, 3584 CS Utrecht, 〈http://www.phil. uu.nl/preprints/lgps/> (2006) 